\title{
Transient Growth Analysis of Compressible Boundary Layers with Parabolized Stability Equations
}

\author{
Pedro Paredes, ${ }^{*}$ Meelan M. Choudhari ${ }^{\dagger}$ Fei Li, ${ }^{\ddagger}$ and Chau-Lyan Chang $§$ \\ NASA Langley Research Center, Hampton, VA 23681
}

\begin{abstract}
The linear form of parabolized linear stability equations (PSE) is used in a variational approach to extend the previous body of results for the optimal, nonmodal disturbance growth in boundary layer flows. This methodology includes the non-parallel effects associated with the spatial development of boundary layer flows. As noted in literature, the optimal initial disturbances correspond to steady counter-rotating streamwise vortices, which subsequently lead to the formation of streamwise-elongated structures, i.e., streaks, via a lift-up effect. The parameter space for optimal growth is extended to the hypersonic Mach number regime without any high enthalpy effects, and the effect of wall cooling is studied with particular emphasis on the role of the initial disturbance location and the value of the spanwise wavenumber that leads to the maximum energy growth up to a specified location. Unlike previous predictions that used a basic state obtained from a self-similar solution to the boundary layer equations, mean flow solutions based on the full Navier-Stokes (NS) equations are used in select cases to help account for the viscous-inviscid interaction near the leading edge of the plate and also for the weak shock wave emanating from that region. These differences in the base flow lead to an increasing reduction with Mach number in the magnitude of optimal growth relative to the predictions based on self-similar meanflow approximation. Finally, the maximum optimal energy gain for the favorable pressure gradient boundary layer near a planar stagnation point is found to be substantially weaker than that in a zero pressure gradient Blasius boundary layer.
\end{abstract}

\section{Nomenclature}

\begin{tabular}{ll}
$G$ & energy gain \\
$M$ & Mach number \\
$\mathbf{M}$ & energy weight matrix \\
$(x, y, z)$ & Cartesian coordinates \\
$(\xi, \eta, \zeta)$ & streamwise, wall-normal and spanwise coordinates in body-fitted coordinate system \\
$h_{1}$ & streamwise metric factor \\
$h_{3}$ & spanwise metric factor \\
$N_{\eta}$ & number of discretization points along the wall-normal direction \\
$\rho$ & density \\
$\nu$ & kinematic viscosity \\
$\gamma$ & heat capacity ratio \\
$T$ & temperature \\
$T_{w}$ & wall temperature \\
$T_{a d}$ & adiabatic wall temperature \\
$R e$ & Reynolds number \\
$R$ & local Reynolds number \\
$(u, v, w)$ & streamwise, wall-normal and spanwise velocity components \\
$\overline{\mathbf{q}}$ & vector of base flow variables \\
\hline${ }^{*}$ NASA NPP Fellow, Computational AeroSciences Branch. AIAA Member \\
${ }_{\dagger}$ Research Scientist, Computational AeroSciences Branch. AIAA Associate Fellow \\
${ }^{\ddagger}$ Research Scientist, Computational AeroSciences Branch. \\
${ }^{\S}$ Research Scientist, Computational AeroSciences Branch. AIAA Member
\end{tabular}




$\begin{array}{ll}\tilde{\mathbf{q}} & \text { vector of perturbation variables } \\ \hat{\mathbf{q}} & \text { vector of amplitude variables } \\ \alpha & \text { streamwise wavenumber } \\ \beta & \text { spanwise wavenumber } \\ L & \text { flate plate characteristic length } \\ \delta & \text { local similarity length scale of boundary layer } \\ \delta .995 & 0.995 \text { boundary layer thickness } \\ \omega & \text { angular frequency } \\ J & \text { objective function } \\ K & \text { kinetic energy norm } \\ E & \text { total energy norm } \\ c & \text { normalization coefficient } \\ \Omega & \text { domain of integration } \\ \mathcal{K} & \text { bilinear concomitant } \\ \mathcal{L} & \text { Lagrangian function }\end{array}$

$\mathbf{A}, \mathbf{B}, \mathbf{C}, \mathbf{D}, \mathbf{L}$ linear matrix operators

$\begin{array}{ll}\text { Subscript } & \\ \text { ad } & \text { adiabatic wall conditio } \\ r & \text { reference value } \\ w & \text { wall condition } \\ 0 & \text { initial disturbance loc } \\ 1 & \text { final optimization locat } \\ \text { Superscripts } & \\ * & \text { dimensional value } \\ \dagger & \text { adjoint } \\ T & \text { transpose } \\ H & \text { conjugate transpose }\end{array}$

Abbreviations

OSE

PSE

Orr-Sommerfeld and Squire Equations

Parabolized Stability Equations

FD-q non-uniform finite difference scheme

NS Navier-Stokes

\section{Introduction}

The most common approach to transition prediction in boundary layer flows relies on the exponential or modal amplification of discrete modes. The classic linear stability theory is mainly concerned with the evolution of individual sinusoidal waves propagating along the boundary layer. The quasi-parallel flow approximation is often used in this context, and it helps reduce the linearized equations of fluid motion to an algebraic eigenvalue problem. In the limit of incompressible flows, the latter problem can be expressed in the form of the Orr-Sommerfeld and Squire equations (OSE). ${ }^{1,2}$ Effects of weak mean-flow non-parallelism can be accounted for by using multiple scale theory (or other similar approaches), ${ }^{3}$ which yields the leading order correction to the local amplification rate and phase speed predicted by the quasi-parallel theory. A more useful extension to the non-parallel stability theory was proposed by Herbert, ${ }^{4}$ who introduced the concept of Parabolized Stability Equations (PSE). Since then, the PSE technique has been applied to a variety of problems, including the linear and nonlinear evolution of instability waves in 2D and 3D shear flows across a broad range of speeds.

Besides the exponential growth characteristics of unstable eigenmodes in a boundary layer flow, the external disturbances, e.g., freestream turbulence and surface roughness, can also have a large influence on the transition process. An additional route to transition may involve nonmodal growth, which refers to situations in which transient growth of disturbance energy is observed even when the flow is modally stable, i.e., all eigenmodes are damped. Mathematically, the transient growth is associated with the non-orthogonality of 
the eigenvectors corresponding to the linear disturbance equations. Physically, the main growth mechanism corresponds to the lift-up effect, ${ }^{5}$ which results from the conservation of horizontal momentum when the fluid particles are displaced vertically, i.e., along the wall-normal direction.

The nonmodal, or equivally transient, growth mechanism has been extensively studied in a large variety of flows. The algebraic growth associated with the inviscid lift-up mechanism was originally identified by Ellingsen \& Palm ${ }^{6}$ for a channel flow. In the 1990s, numerous temporal nonmodal growth studies of incompressible, two-dimensional, parallel shear flows appeared, among others by Butler \& Farrell, ${ }^{7}$ Reddy \& Henningson ${ }^{8}$ and Trefethen et al. ${ }^{9}$ Lately, Hanifi et al. ${ }^{10}$ included the compressibility effects in their study of temporal, nonmodal transient growth in zero-pressure-gradient flat-plate boundary layers. The first spatial analyses of nonmodal growth in a Blasius boundary layer were presented by Andersson et al. ${ }^{11}$ and Luchini, ${ }^{12}$ who used the linearized boundary layer equations (or boundary region equations ${ }^{13-15}$ ), which include the non-parallel effects associated with boundary layer development and the viscous diffusion of the disturbance field in both wall-normal and spanwise directions. The nonmodal analysis of compressible boundary layers was continued by Tumin \& Reshotko, ${ }^{16}$ who reformulated the temporal analysis of Hanifi et al. ${ }^{10}$ in a spatial framework, but still assuming the parallel flow approximation. The non-parallel effects were included in their subsequent publications ${ }^{17-20}$ by solving a parabolic set of equations based on the boundary layer approximation. These authors also addressed the effects of convex surface curvature by studying optimal growth in the boundary layer over a sphere. ${ }^{18,19}$ They concluded that increasing convex curvature reduces nonmodal growth. These studies also considered the effects of wall cooling and concluded that reducing the wall temperature increases the nonmodal growth. Zuccher et al. ${ }^{20}$ studied transient growth in the boundary layer over a sharp circular cone at zero angle of attack and freestream Mach number of 6 . The basic state was based on the self-similar solution to boundary layer equations. The edge Mach number was still supersonic, i.e., $M<4.5$, because of the selected half-angle of the cone. The inflow location was selected far downstream from the cone tip $\left(x_{0} / x_{1}>0.2\right)$ to avoid interaction with the shock wave. As might be expected, the computed transient growth characteristics collapse onto those for the flat plate boundary layer with the same edge Mach number as $x_{0} \rightarrow x_{1}$ and the azimuthal wavenumber becomes large (because the effects of geometric divergence and surface curvature become small in these two limits). They reported that the main effect of the flow divergence over the circular cone and sphere is the presence of an optimal downstream location in which a maximum energy gain is reached for a fixed initial disturbance position. Strong similarities between compressible and incompressible cases led to the conclusion that at least up to supersonic Mach numbers, both speed regimes exhibit the same physical mechanism of nonmodal growth, i.e., the lift-up effect.

Recently, transient growth has been suggested as a candidate mechanism for several cases of bypass transition. ${ }^{21}$ The latter has been historically used to differentiate the transition phenomena that are not fully understood on a theoretical basis from the well known paths to transition. ${ }^{22}$ Examples of transition often classified as bypass transition include the subcritical transition observed in Poiseuille pipe flow experiments, ${ }^{23,24}$ transition due to distributed surface roughness on flat plates ${ }^{25,26}$ or cones, ${ }^{27}$ and subcritical transition observed on spherical forebodies. ${ }^{28-31}$ However, the existing work on transient growth is limited to boundary layer flows with subsonic or supersonic values of the edge Mach number; and no studies of optimal growth in hypersonic boundary layers are known to the authors.

This paper extends the previous body of results for the optimal, nonmodal disturbance growth in boundary layer flows in several ways. Section II provides a summary of the optimal growth theory based on the PSE. Validation of numerics against previous results for $M=0$ and $M=3$ is presented in Section III. The existing results are extended to a realistic basic state that is based on the full Navier-Stokes (NS) equations and, hence, accounts for both the viscous-inviscid interaction near the leading edge of the plate and the weak shock wave emanating from that region. The basic states obtained in this manner are used to study optimal transient growth at four specific flow conditions: $M=3$ with adiabatic wall, $M=6$ with $T_{w} / T_{a d}=0.4$ and 0.8 , and $M=10$ with $T_{w} / T_{a d}=0.35$. Effects of wall cooling and Mach number are also examined using both self-similar and NS basic states. Additionally, the effect of non-zero pressure gradient is taken into account by performing the transient growth analysis of the incompressible, planar stagnation point boundary layer flow, i.e., the Hiemenz flow, in Section IV. Summary and conclusions are presented in Section V.

As pointed out by Choudhari and Fischer, ${ }^{32}$ it is important to model the transient growth phenomenon as an inhomogeneous boundary value problem associated with a physically realizable forcing environment ${ }^{33}$ rather than via the optimal growth formulation alone. However, the issue of realizable transient growth must be addressed on a case by case basis. The results presented in the present work provide a useful starting 
point for such investigations in the context of high Mach number flows.

\section{Methodology}

The variational formulation underlying optimal growth predictions based on the parabolized stability equations is well explained in the literature. ${ }^{34-36}$ The method is outlined here for completeness. There are strong similarities with the optimization approach based on the linearized boundary layer equations. ${ }^{11,12,17}$ The advantage of the PSE based formulation is that it may be used for more complex base flows where the flow evolves slowly along the streamwise direction but the boundary layer approximation may not hold and that it can be easily extended to unsteady disturbances. Ref. 37 provides a detailed comparison between both formulations for incompressible flows. While infinite Reynolds number asymptotic results cannot be directly computed using the PSE, good agreement is achieved between the two methodologies for incompressible and compressible regimes as shown in Section III.

\section{II.A. Governing Equations}

In the linear PSE context, the perturbations have the form

$$
\tilde{\mathbf{q}}(\xi, \eta, \zeta, t)=\hat{\mathbf{q}}(\xi, \eta) \exp \left[\mathrm{i}\left(\int_{\xi_{0}}^{\xi} \alpha\left(\xi^{\prime}\right) \mathrm{d} \xi^{\prime}+\beta \zeta-\omega t\right)\right]+\text { c.c., }
$$

where c.c. denotes complex conjugate. The suitably-nondimensionalized, orthogonal, curvilinear coordinate system $(\xi, \eta, \zeta)$ denotes the steamwise, spanwise, and wall-normal coordinates, respectively, and $(u, v, w)$ represent the corresponding velocity components. The vector of perturbation fluid variables is denoted by $\tilde{\mathbf{q}}(\xi, \eta, \zeta, t)=(\tilde{\rho}, \tilde{u}, \tilde{v}, \tilde{w}, \tilde{T})^{T}$, and the vector of respective amplitude functions is $\hat{\mathbf{q}}(\xi, \eta)=(\hat{\rho}, \hat{u}, \hat{v}, \hat{w}, \hat{T})^{T}$. The streamwise and spanwise wavenumbers are $\alpha$ and $\beta$, respectively; and $\omega$ is the angular frequency of the perturbation.

Upon introduction of the perturbation form (1) into the linearized NS equations together with the assumption of a slow streamwise dependence of the basic state and the amplitude functions, thus neglecting the viscous derivatives in $\xi$, the PSE are recovered as follows

$$
\left(\mathbf{A}+\mathbf{B} \frac{\partial}{\partial \eta}+\mathbf{C} \frac{\partial^{2}}{\partial \eta^{2}}+\mathbf{D} \frac{1}{h_{1}} \frac{\partial}{\partial \xi}\right) \hat{\mathbf{q}}(\xi, \eta)=0 .
$$

The linear operators $\mathbf{A}, \mathbf{B}, \mathbf{C}$ and $\mathbf{D}$ are given by Pralits ${ }^{38}$ and $h_{1}$ is the metric factor associated with the streamwise curvature. The system of equations (2) is not fully parabolic due to the term $\partial \hat{p} / \partial \xi$ in the streamwise momentum equation. ${ }^{39-43}$ However, for the purely stationary disturbances of interest in this work, this term can be dropped from the equations as justified by Refs. 35 and 44, who found that the term, $\partial \hat{p} / \partial \xi$, is of higher order for transient growth problems, and can be neglected without any loss of accuracy.

\section{II.B. Optimality System}

The optimal initial disturbance, $\tilde{\mathbf{q}}_{0}$, is defined as the initial (i.e., inflow) condition at $\xi_{0}$ that experiences the maximum energy amplification up to a specified position, $\xi_{1}$. To determine $\tilde{\mathbf{q}}_{0}$, the following objective function needs to be maximized,

$$
J(\tilde{\mathbf{q}})=\frac{E\left(\xi_{1}\right)}{E\left(\xi_{0}\right)},
$$

where $E$ denotes the energy norm of $\tilde{\mathbf{q}}$,

$$
E(\xi)=\int_{\zeta} \int_{\eta} \tilde{\mathbf{q}}(\xi)^{H} \mathbf{M} \tilde{\mathbf{q}}(\xi) \mathrm{d} \eta \mathrm{d} \zeta .
$$

Here, $\mathbf{M}$ is the energy weight matrix; and the superscript $H$ denotes the conjugate transpose of a vector or matrix quantity. The choice of the energy norm would have an effect on the results. Here, the energy norm proposed by Mack, ${ }^{45}$ and Hanifi et al. ${ }^{10}$ is used and is defined by choosing

$$
\mathbf{M}=\operatorname{diag}\left[\frac{\bar{T}(\xi)}{\gamma \bar{\rho}(\xi) M^{2}}, \bar{\rho}(\xi), \bar{\rho}(\xi), \bar{\rho}(\xi), \frac{\bar{\rho}(\xi)}{\gamma(\gamma-1) \bar{T}(\xi) M^{2}}\right] .
$$


The disturbance that maximizes the objective function $J(\tilde{\mathbf{q}})$ is calculated using the Lagrange functional

$$
\mathcal{L}\left(\tilde{\mathbf{q}}, \tilde{\mathbf{q}}^{\dagger}\right)=J(\tilde{\mathbf{q}})-\left\langle\tilde{\mathbf{q}}^{\dagger}, \mathbf{L} \tilde{\mathbf{q}}\right\rangle,
$$

where the inner product is defined as

$$
\langle a, b\rangle=\int_{\Omega} a^{H} b \mathrm{~d} \Omega,
$$

L $\tilde{\mathbf{q}}$ represents the left-hand side of the stability equation $(2)$, and $\tilde{\mathbf{q}}^{\dagger}=\left(\tilde{\rho}^{\dagger}, \tilde{u}^{\dagger}, \tilde{v}^{\dagger}, \tilde{w}^{\dagger}, \tilde{T}^{\dagger}\right)^{T}$ is the Lagrange multiplier, i.e., the vector of adjoint disturbance variables. The roots of the first variation of the Lagrange functional (6) represent the optimal disturbance and its corresponding adjoint. Setting the gradient of $\mathcal{L}$ with respect to $\tilde{\mathbf{q}}^{\dagger}$ to zero, i.e., $\nabla_{\tilde{\mathbf{q}}^{\dagger}} \mathcal{L}=0$, leads to the linear stability equations, $\mathbf{L} \tilde{\mathbf{q}}=0$. To set the second gradient with respect to $\tilde{\mathbf{q}}$ to zero, i.e., $\nabla_{\tilde{\mathbf{q}}} \mathcal{L}=0$, the following identity is used,

$$
\left\langle\tilde{\mathbf{q}}^{\dagger}, \mathbf{L} \tilde{\mathbf{q}}\right\rangle=\left\langle\mathbf{L}^{\dagger} \tilde{\mathbf{q}}^{\dagger}, \tilde{\mathbf{q}}\right\rangle+\int_{\Omega} \nabla \cdot \mathcal{K}\left(\tilde{\mathbf{q}}, \tilde{\mathbf{q}}^{\dagger}\right) \mathrm{d} \Omega
$$

which is derived by expanding $\left\langle\tilde{\mathbf{q}}^{\dagger}, \mathbf{L} \tilde{\mathbf{q}}\right\rangle$ by using integration by parts. Here, the adjoint equations are defined as $\mathbf{L}^{\dagger} \tilde{\mathbf{q}}^{\dagger}=0$, and $\mathcal{K}$ is known as the bilinear concomitant, which represents the boundary terms in the three directions. Then, to accomplish $\nabla_{\tilde{\mathbf{q}}} \mathcal{L}=0$, the adjoint equations, $\mathbf{L}^{\dagger} \tilde{\mathbf{q}}^{\dagger}=0$, must be satisfied, as well as the optimality conditions at $\xi_{0}$ and $\xi_{1}$, which result from the streamwise boundary terms, $\mathcal{K}_{\xi}$, and the gradient of the objective function, $\nabla_{\tilde{\mathbf{q}}} J$, when the boundary conditions for the direct and adjoint variables are chosen such that the wall-normal and spanwise boundary terms, $\mathcal{K}_{\eta}$ and $\mathcal{K}_{\zeta}$, respectively, are zero. The optimality conditions result

$$
\mathbf{D}^{H} \tilde{\mathbf{q}}_{k}^{\dagger}-c_{k} \mathbf{M} \tilde{\mathbf{q}}_{k}=0
$$

where the subscript $k$ is 0 or 1 denoting either $\xi_{0}$ or $\xi_{1}$, and $c_{k}$ denotes a normalization coefficient that adjusts $E_{0}=E\left(\xi_{0}\right)=1$. Thus, the initial condition of the adjoint equations or backward marching integration at $\xi_{1}$ can be written as

$$
\tilde{\mathbf{q}}_{1}^{\dagger}=\left(\mathbf{D}^{H}\right)^{-1} c_{1} \mathbf{M}_{1} \tilde{\mathbf{q}}_{1} ;
$$

and the optimal initial condition for the direct equations or forward marching integration can be written as

$$
\tilde{\mathbf{q}}_{0}=\frac{1}{c_{0}}\left(\mathbf{M}_{0}\right)^{-1} \mathbf{D}^{H} \tilde{\mathbf{q}}_{0}^{\dagger} .
$$

Note that $\mathbf{D}^{H}$ is singular at the wall and that the adjoint equations do not comprise boundary conditions for $\tilde{\rho}^{\dagger}$ at the wall. The value at the wall for $\tilde{\rho}_{1}^{\dagger}$ is obtained from the wall-normal equation. Additional assumptions need to be considered for the optimal initial condition, because the optimality condition for the streamwise velocity becomes

$$
\tilde{u}\left(\xi_{0}\right)=\tilde{\rho}^{\dagger}\left(\xi_{0}\right)+\bar{u}\left(\xi_{0}\right) \tilde{u}^{\dagger}\left(\xi_{0}\right) .
$$

Because $\tilde{\rho}^{\dagger}\left(\xi_{0}\right)$ is non-zero at the wall, the equation (12) yields a non-zero value of $\tilde{u}\left(\xi_{0}\right)$ at the wall. Tempelmann et al. ${ }^{36}$ found that neglecting $\tilde{\rho}^{\dagger}\left(\xi_{0}\right)$ is the best option to satisfy both equation (12) and the no-slip boundary condition, and leads to results that are identical to other approaches. ${ }^{19}$

The optimality system is solved in an iterative manner, starting from a random solution at $\xi_{0}$, which must satisfy the boundary conditions. The PSE, L $\tilde{\mathbf{q}}=0$, are used to integrate $\tilde{\mathbf{q}}$ up to $\xi_{1}$, where the optimality condition (12) is used to obtain the initial condition for the backward adjoint PSE integration, $\mathbf{L}^{\dagger} \tilde{\mathbf{q}}^{\dagger}=0$. At $\xi_{0}$, the adjoint solution is used to calculate the new initial condition for the forward PSE integration by using equation (11). The iterative procedure is terminated when the objective function, $J=G$, is converged up a certain tolerance, which was set equal to $10^{-4}$ in the present computations.

\section{Optimal Gain in Zero-Pressure-Gradient Boundary Layers}

Transient growth in zero-pressure-gradient flat plate boundary layer flow is studied first for selected Mach numbers. For this problem, the computational and physical coordinates coincide, i.e., $(\xi, \eta, \zeta) \equiv(x, y, z)$. The self-similar scale proportional to boundary layer thickness is $\delta=\sqrt{\nu^{*} x^{*} / u_{r}^{*}}$, where subscript $r$ denotes the reference values and the superscript $*$ indicates dimensional values. Because the streamwise length scale of the disturbance undergoing transient growth corresponds to the distance $x_{1}^{*}-x_{0}^{*}$, which is assumed to be 
of $\mathcal{O}\left(x_{1}^{*}\right)$, the streamwise coordinate $x^{*}$ is scaled by $x_{1}^{*}=L$; and the other two coordinates are scaled with respect to the value of $\delta$ at $x_{1}^{*}=L$, i.e., the location selected to maximize the transient growth relative to $x_{0}^{*}$. Therefore, the Reynolds number introduced into the equations becomes $R e=\sqrt{R e_{L}}$. The streamwise location in terms of the local Reynolds number is $R=R e_{\delta}=\sqrt{x^{*} u_{r}^{*} / \nu^{*}}$. Previous results ${ }^{11,12,17}$ using the self-similar boundary layer solution as basic state have shown that in the limit of large Reynolds numbers the optimal gain $G$ scales with the body length Reynolds number $R e_{L}$. However, similar results are also found for lower $R e_{L} \cdot{ }^{11,17}$

Finite differences ${ }^{46,47}$ (FD-q) of eighth order are used for discretization of the PSE along the wall-normal coordinate. The discretized PSE are integrated along the streamwise coordinate by using second-order backward differentiation. The number of discretization points in both directions was varied in selected cases to ensure convergence of the optimal gain predictions. The wall-normal direction was discretized using $N_{y}=141$ for $M=10^{-3}$ to as high as $N_{y}=401$ for $M=10$. The nodes are clustered towards the wall. ${ }^{47} \mathrm{~A}$ non-constant step along the streamwise direction was used, corresponding to a constant increment of $\Delta R$. No-slip, isothermal boundary conditions are used at the wall, i.e., $\hat{u}=\hat{v}=\hat{w}=\hat{T}=0$. The isothermal boundary conditions may not hold for all flow conditions. However, the results appear to be insensitive to the thermal boundary condition at the surface. Therefore, a conjugate heat transfer analysis is deemed unnecessary for the purpose of this paper. The amplitude functions are forced to decay at the farfield boundary by imposing the Dirichlet conditions $\hat{\rho}=\hat{u}=\hat{w}=\hat{T}=0$. We choose not to constrain the inflow perturbation profiles to be identically zero within the freestream region outside of the leading edge shock. If the source of the inflow disturbance were to be at the surface, then a condition of this type would have been appropriate. However, the physical origin of the inflow disturbance is left open in the present optimal growth formulation, and we simply impose the condition that the inflow profiles become zero at infinity. Interaction of the weak but nonzero freestream perturbation with the basic state shock is treated in the spirit of a shock capturing calculation.

\section{III.A. Code Validation}

First, we compare the transient growth predictions based on the PSE formulation with those based on the large Reynolds number asymptotic framework that leads to linearized boundary layer equations. ${ }^{11,17}$ To allow meaningful comparison with the asymptotic framework, a large value of the Reynolds number parameter $\left(R e_{L}=10^{8}\right)$ is used.

Incompressible results by Andersson et al. ${ }^{11}$ are reproduced by setting $M=10^{-3}$ and $x_{0}=0$. Figure 1 (a) shows the optimal energy gain as a function of the spanwise wavenumber. Excellent agreement with the results of Andersson et al. ${ }^{11}$ is observed. Results of Tumin \& Reshotko ${ }^{17}$ at a supersonic Mach number of 3 are used for the next comparison. Figure 1(b) shows the optimal gain relative to $x_{0}=0$ for three different wall temperature ratios. Again an excellent agreement is noted in all cases.

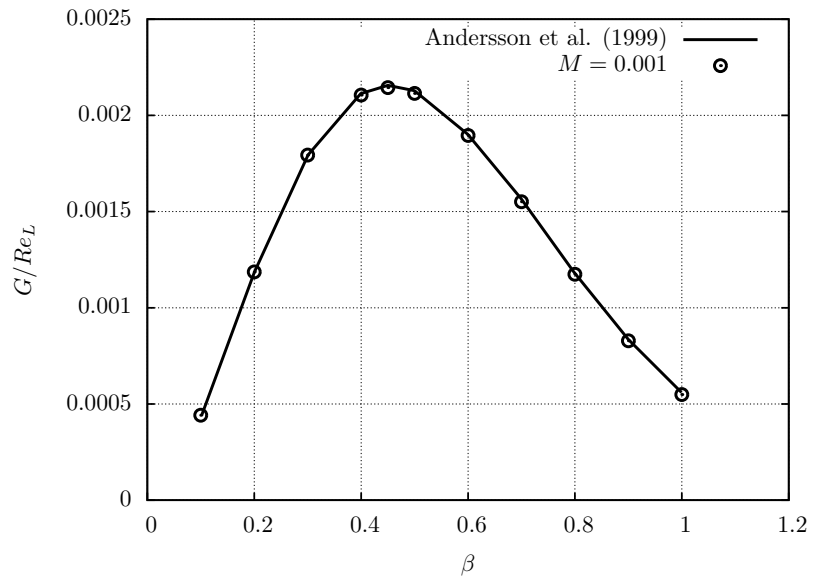

(a) $M=10^{-3}$

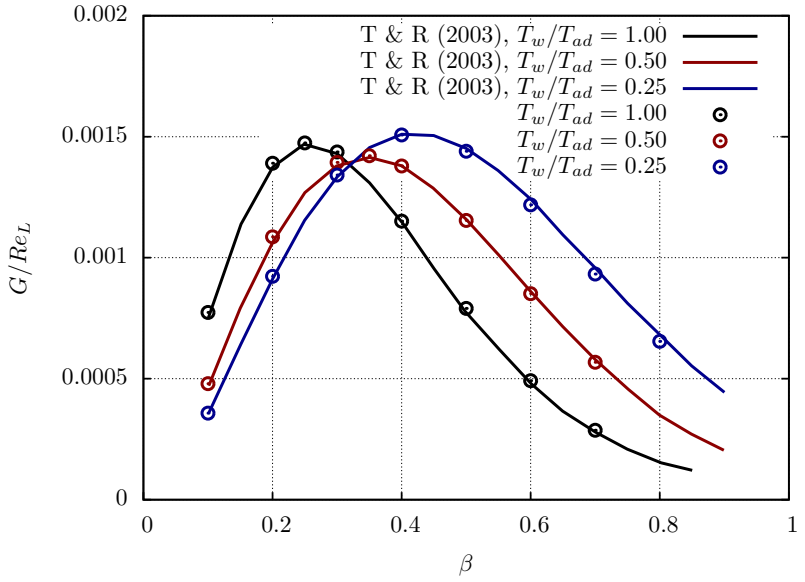

(b) $M=3$

Figure 1. Comparison with optimal gain predictions by (a) Andersson et al. ${ }^{11}$ for an incompressible flat plate boundary layer setting $M=10^{-3}$, and by (b) Tumin \& Reshotko ${ }^{17}$ for a Mach 3 flat plate boundary layer for selected wall temperature ratios $\left(R e_{L}=10^{8}, T_{0}^{d}=333 \mathrm{~K}\right)$. 


\section{III.B. Effect of Weak Leading-Edge Shock on Transient Growth}

The previous analyses of transient growth in compressible boundary layers ${ }^{17,19}$ are extended by increasing the Mach number up to 10 and by using a basic state definition based on the NS equations in lieu of the self-similar solutions to boundary layer equations that were used in the previous studies at lower Mach numbers. ${ }^{17,19}$ The NS mean flow accounts for both the viscous-inviscid interaction near the leading edge and the weak shock wave emanating from that region.

The mean boundary layer flows over the flat plate configurations were computed on various grids with a second-order accurate algorithm (based on the Low Diffusion Flux Splitting Scheme) as implemented in the finite-volume, structured grid, compressible NS flow solver VULCAN. ${ }^{48}$, a The weak shock emanating from the leading edge of the plate was captured within the computational domain. Even though the shock is relatively weak in comparison with a normal shock at the same incoming Mach number, the computational mesh was approximately aligned with the shock surface. Given the very low Reynolds number near the leading edge (unit Reynolds number based on the freestream velocity and leading edge radius), the shock layer is highly viscous and the flow gradients are smeared across the shock layer. At sufficiently large distances (compared with the leading edge radius), the shock becomes relatively sharper but also weaker.

The nose radius is set to $r_{n}=1 \mu \mathrm{m}$. The free stream unit Reynolds number is set to $R e^{\prime}=10^{6} / \mathrm{m}$. The NS solution for a flat plate boundary layer was obtained for four flow conditions, namely, $M=3$ with adiabatic wall, $M=6$ with $T_{w} / T_{a d}=0.4$ and 0.8 , and $M=10$ with $T_{w} / T_{a d}=0.35$. Figure 2 (a) shows iso-contours of the wall-normal velocity in the vicinity of the leading edge. The effects of viscous-inviscid interaction and the shock wave lead to a deviation from the self-similar boundary layer profiles, as observed in figure 2(b). The NS profiles match the self-similar solution, except for $R=20$. Figure 3 shows a similar comparison for the Mach $6\left(T_{w} / T_{a d}=0.8\right)$ and Mach $10\left(T_{w} / T_{a d}=0.35\right)$ cases. The increased Mach number produces a stronger deviation from the self-similar profile near the leading edge. Although not shown, the streamwise velocity and temperature profiles converge to the self-similar solution at $R \geq 100$ after the profiles are normalized by the corresponding values at the boundary layer edge.

Because the shock is fairly weak and the wall-normal computational grid is rather fine, no difficulties were encountered during disturbance calculations that included the basic state shock within the computational domain. In other words, no special treatment to address the disturbance interaction with the shock was found necessary for the calculations presented in this paper. Grid sensitivity analysis was performed for the Mach 10 case by using three grids with successively increasing resolutions. The baseline grid had 1,793 points in the streamwise directions and 513 points along the wall-normal axis. The other two grids were coarser and finer, respectively, by a factor of two in each direction. The grid distribution was identical across all three grids, so that the grid convergence process was parametrized by a single parameter corresponding to the overall cell count. Excellent agreement was found among the mean flow profiles obtained from all three grids as well as among the transient growth prediction based on these grids.

Transient growth results corresponding to a Mach 3, adiabatic boundary layer obtained from the NS equations are shown in figure 4 (a) for $R_{1}=1,000$ as the final optimization position. Although not shown here, results for $R_{0} / R_{1}=0.01$ (i.e., $x_{0} / x_{1}=0.0001$ ), which needed a much higher resolution due to the clustering of points inside the boundary layer, proved that the gain factors for $R_{1} / R_{0}=0.02$ have reached their asymptote corresponding to $R_{0} / R_{1} \rightarrow 0$. Figure $4(\mathrm{~b})$ shows a maximum $9 \%$ reduction of optimal energy gain between self-similar and NS basic states when the initial optimization position is located near the leading edge. For the same conditions, i.e., Mach 3, adiabatic boundary layer with $R_{0}=20, R_{1}=1,000$ and $\beta=0.25$, the initial optimal and final perturbations are compared for the self-similar and NS basic states, respectively, in figure 5. The shape of the perturbation at the final station is almost identical for both basic states. The differences between the initial optimal perturbations are more noticeable. As seen from the $R_{0}=20$ basic state profiles in figure 2(b), the weak shock layer is located just above the boundary layer edge at the inflow location. The presence of the shock in the basic state profiles leads to a strong gradient in the disturbance variables, which decay faster in the free stream above the shock for the NS basic state case. As previously mentioned, the weak leading-edge shock wave decreases the growth of the optimal perturbation. The reduction in energy gain is also apparent from the comparison of the disturbance profiles at $R=R_{1}$ for both basic states. The maximum values of $\hat{T}, \hat{\rho}$, and $\hat{u}$ are smaller for the NS basic state case.

Figure 6 shows optimal gain results for Mach 6 boundary layers with $T_{w} / T_{a d}=0.4$ and 0.8 . The basic state solutions are obtained by solving the NS equations. Comparison of the optimal gain magnitudes from

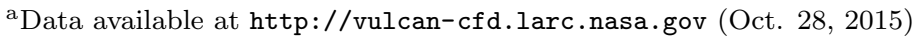




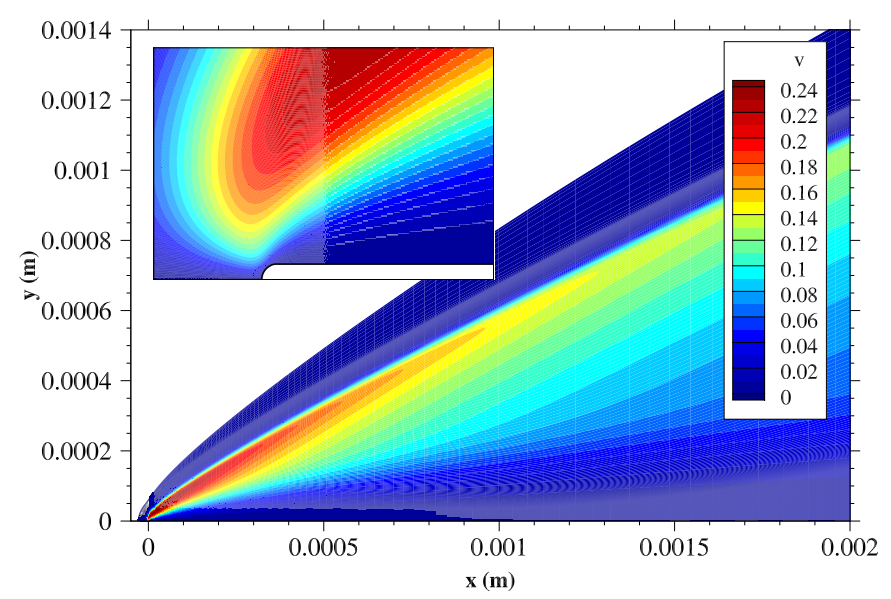

(a) $\bar{v}$ velocity of NS mean flow

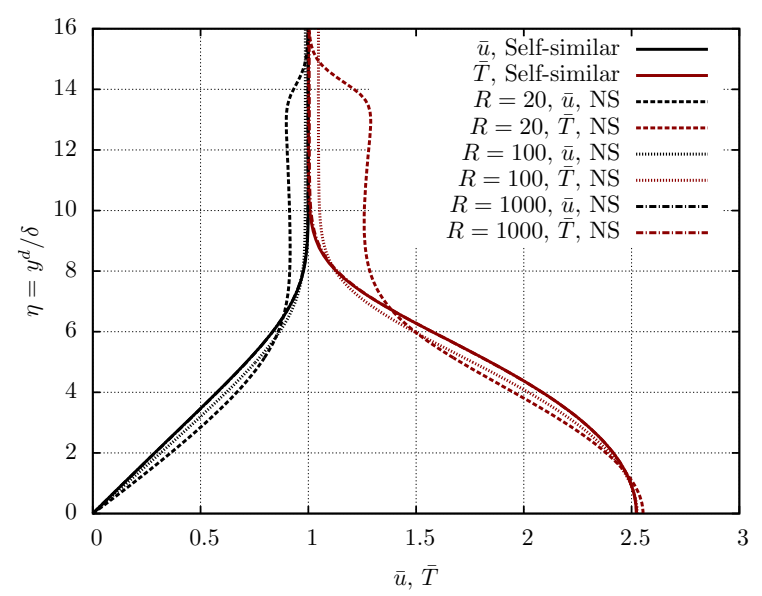

(b) Comparison with self-similar profiles

Figure 2. (a) Iso-contours of $\bar{v}$ velocity of NS solution for an adiabatic Mach 3 boundary layer, including a zoom of the leading edge zone. (b) Comparison of self-similar and NS solution profiles for selected streamwise positions. The profiles at $R=20$ correspond to a streamwise location of $x=0.0004 \mathbf{m}$ in part (a).

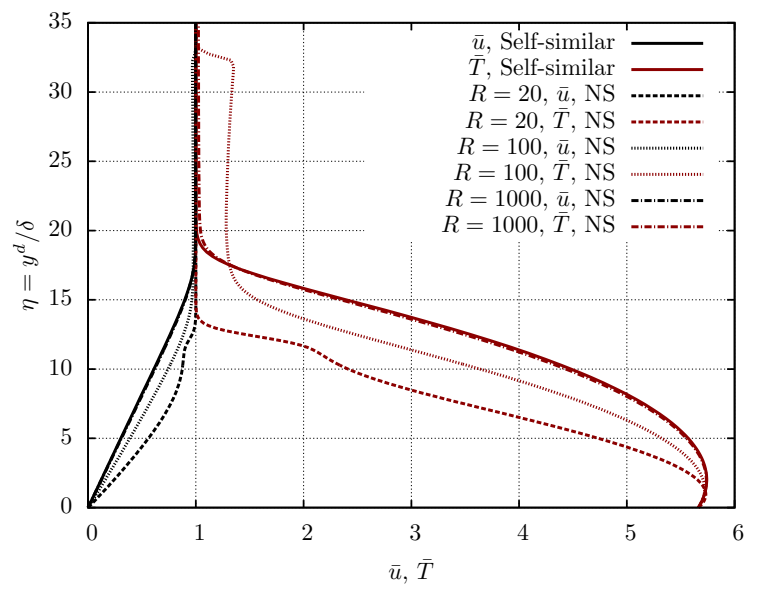

(a) $M=6, T_{w} / T_{a d}=0.8$

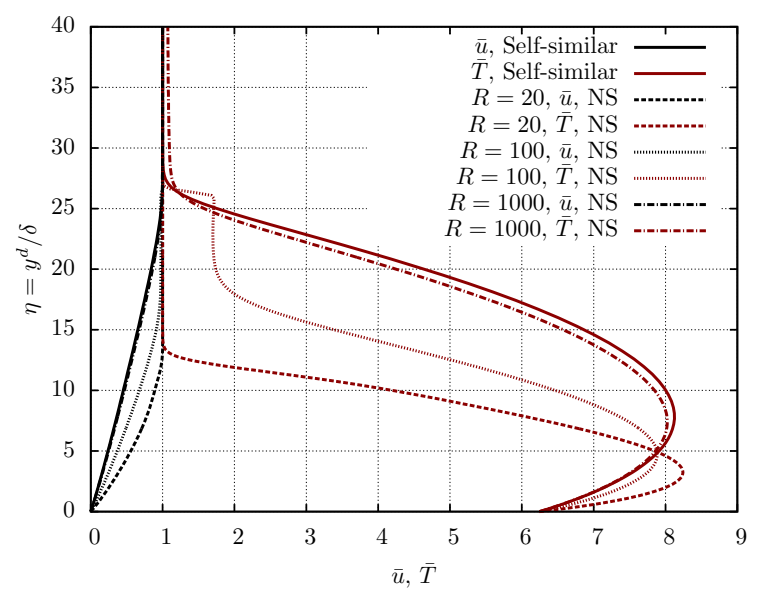

(b) $M=10, T_{w} / T_{a d}=0.35$

Figure 3. Comparison of self-similar and NS solution profiles for a Mach $6\left(T_{w} / T_{a d}=0.8\right)$ and a Mach $10\left(T_{w} / T_{a d}=0.35\right)$ boundary layers at selected streamwise positions.

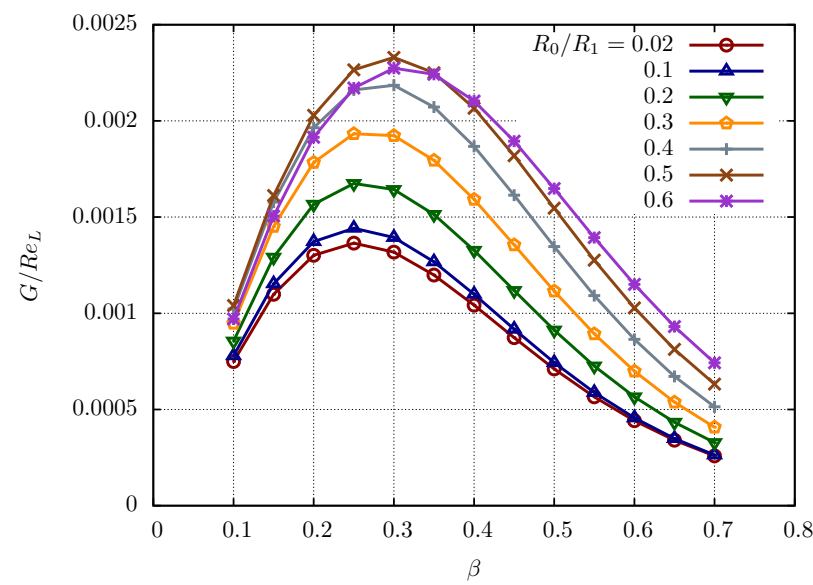

(a) Effect of $x_{0}$ on optimal energy gain

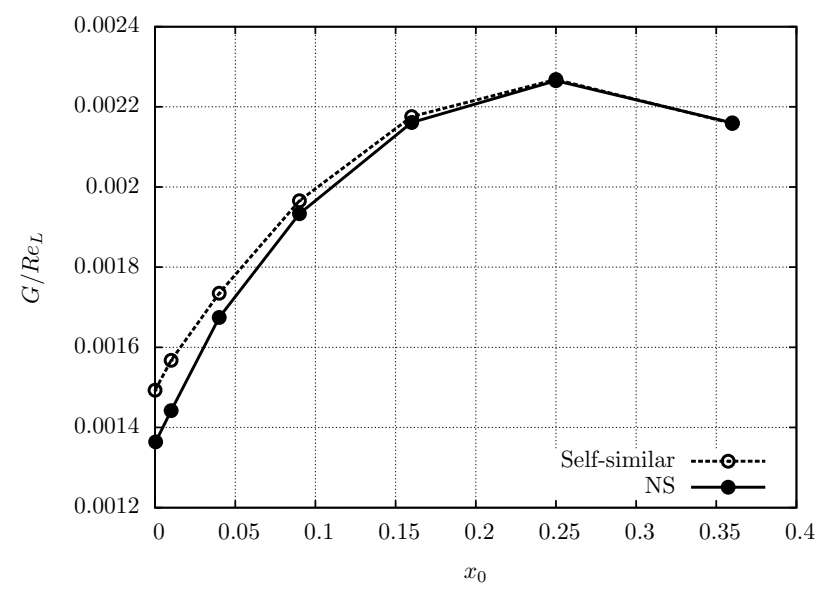

(b) Comparison with self-similar (SS) basic state with $\beta=0.25$

Figure 4. (a) Effect of initial optimization position, $R_{0}$, in the optimal energy gain with $R_{1}=1,000$ and the NS basic state of flat plate boundary layer at freestream Mach number of 3. (b) Comparison of optimal energy gain using the self-similar (SS) and NS basic states for $\beta=0.25$. 


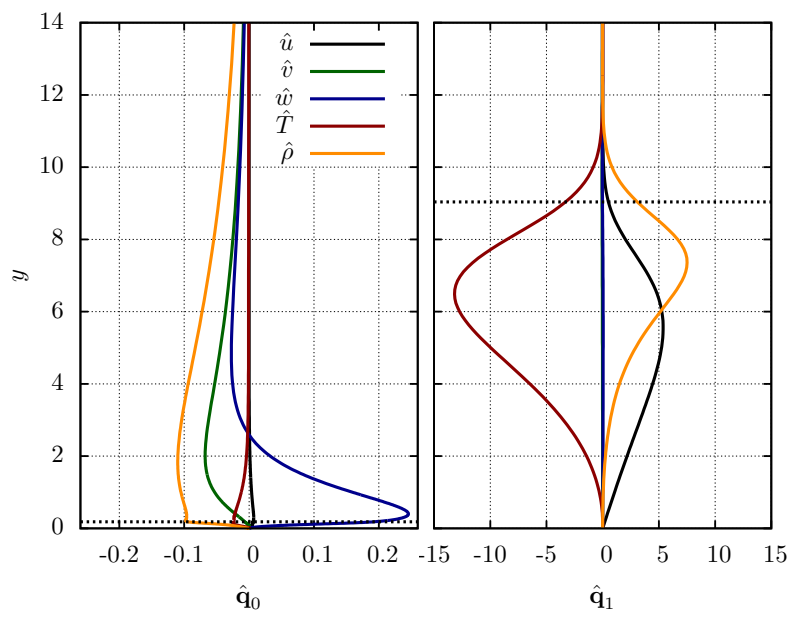

(a) Self-similar basic state

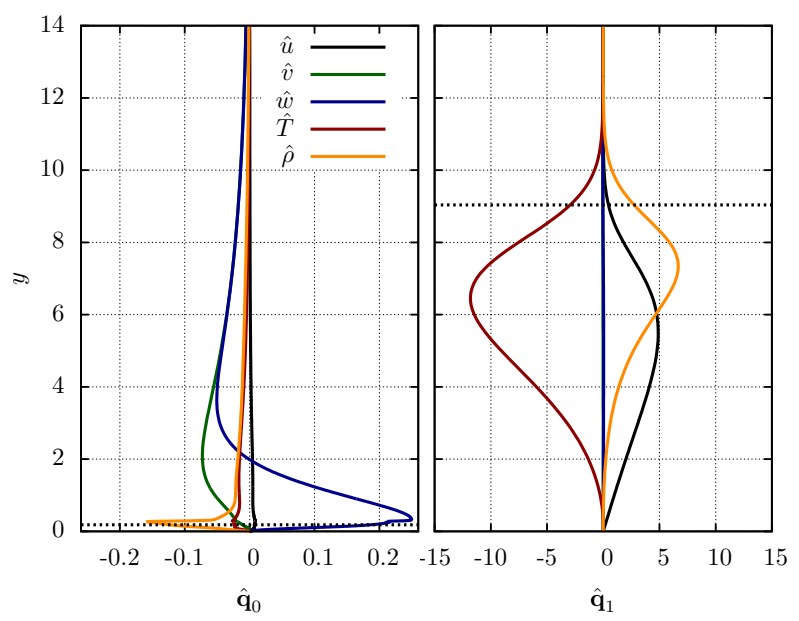

(b) NS basic state

Figure 5. Initial and final amplitude vectors with $R_{0}=20$ and $R_{1}=1,000$ and $\beta=0.25$ using both the self-similar and NS basic states of Mach 3 adiabatic flat plate boundary layer. The dashed lines denote the position of the boundary layer edge, i.e., $\bar{u}=0.995$.

figures $6(\mathrm{a})$ and $6(\mathrm{~b})$ shows that, similar to previous studies of transient growth at lower mach numbers, ${ }^{17,19}$ wall cooling enhances the nonmodal growth in hypersonic boundary layers as well. The effect of wall-cooling will be thoroughly studied in Subsection III.C below. The effect of initial disturbance location and the optimization location downstream and is studied in figures 6 (a) and 6 (b) by varying $R_{1} / R_{0}$, while keeping $R_{1}$ fixed at $R_{1}=1,000$ and $R_{1}=2,000$ for $T_{w} / T_{a d}=0.4$ and $T_{w} / T_{a d}=0.8$, respectively. The effect of the shock layer emanating from the leading edge is in-line with the Mach 3 results discussed earlier. Because of the stronger shock at $M=6$, the shock-induced reduction in optimal gain for initial locations near the leading edge is larger than that at $M=3$. Also, the effect is greater for the $T_{w} / T_{a d}=0.8$ because the boundary layer thickness is larger for wall temperature closer to the adiabatic temperature. For both conditions, i.e., $T_{w} / T_{a d}=0.4$ and 0.8 , the maximum energy gain is achieved for $R_{0} / R_{1}=0.5$. Transient growth results with $R_{0} / R_{1}=0.1$ and $R_{1}=1,000$ could not be recovered by using the present technique. The presence of a stronger inviscid-viscous interaction zone, together with the stronger shock wave near the leading edge, prevents the computation of converged results for initial locations closer to the leading edge, $R_{0}<200$. Because the highest optimal gain occurs for inflow locations well downstream of the leading edge $\left(R_{0} / R_{1} \approx 0.5\right)$, the limitation to a nonzero but small $R_{0} / R_{1}$ was deemed acceptable for the purpose of the present study.

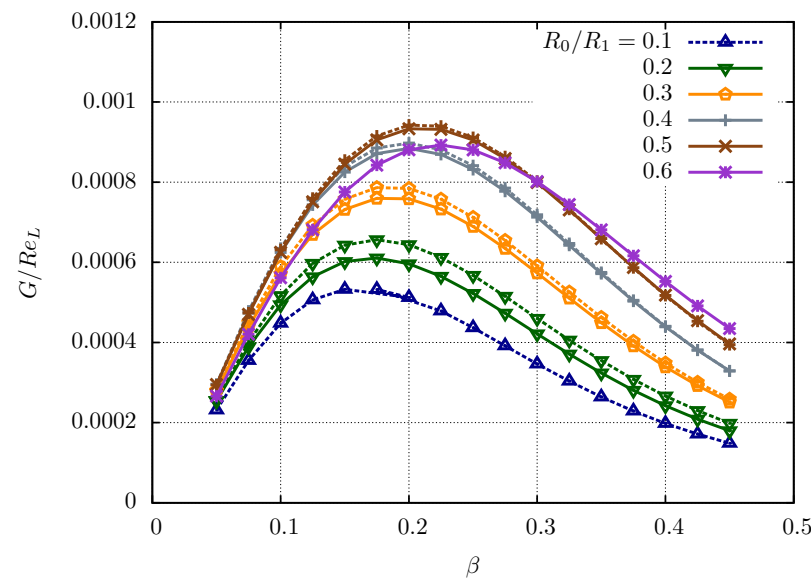

(a) $M=6, T_{w} / T_{a d}=0.4$

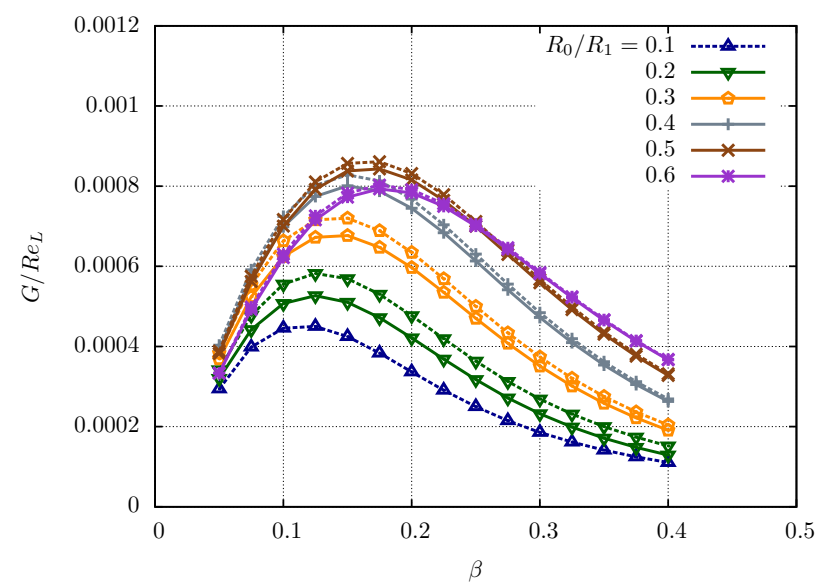

(b) $M=6, T_{w} / T_{a d}=0.8$

Figure 6. Effect of initial optimization position, $R_{0}$, with $R_{1}=1,000$ (solid lines) and $R_{1}=2,000$ (dashed lines), in the optimal energy gain using the NS basic states of flat plate boundary layers at freestream Mach number of 6 and (a) $T_{w} / T_{a d}=0.4$ and (b) $T_{w} / T_{a d}=0.8$. 
The evolution of the disturbance vector along the Mach 6 boundary layer with $T_{w} / T_{a d}=0.8$ is shown in figure 7 with initial disturbance location close to the leading edge $\left(x_{0} / x_{1}=0.01\right.$, i.e., $R_{0} / R_{1}=0.1$, along with $R_{1}=2,000$, and $\beta=0.116$ ) and in figure 8 with initial position that leads to maximum energy growth at $R_{1}=1,000\left(R_{0} / R_{1}=0.5\right.$ and $\left.\beta=0.166\right)$. For the first case $\left(R_{0} / R_{1}=0.1\right)$, the main component of the initial optimal perturbation (figure $7(\mathrm{a})$ ) is the spanwise velocity $\hat{w}$ as also observed in figure 5 for the Mach 3 case. The peak of $\hat{w}$ occurs just below the boundary layer edge. The peaks of $\hat{T}, \hat{\rho}$, and $\hat{u}$ are located in a similar position relative to the boundary layer edge during the evolution of the optimal initial disturbance, indicating a similar disturbance shape increasing in magnitude along the streamwise coordinate. Figure 8 (a) shows the optimal initial perturbation for $R_{0} / R_{1}=0.5$. In this case, the energy of the initial optimal perturbation is more evenly distributed between the wall-normal and spanwise velocity components, $\hat{v}$ and $\hat{w}$.

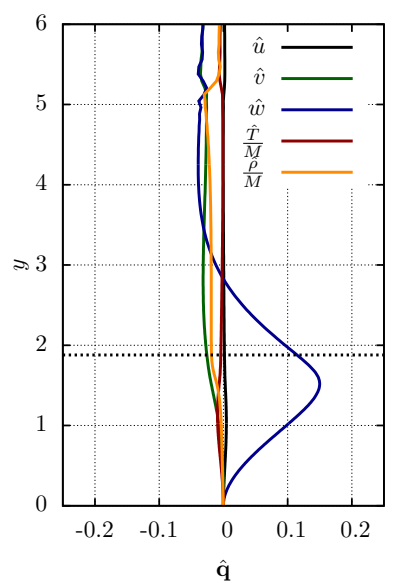

(a) $R / R_{1}=0.1$

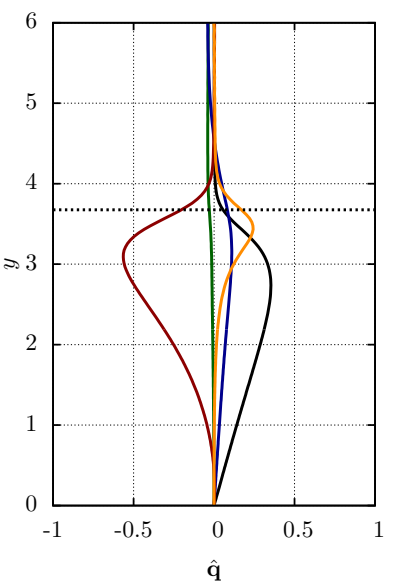

(b) $R / R_{1}=0.2$

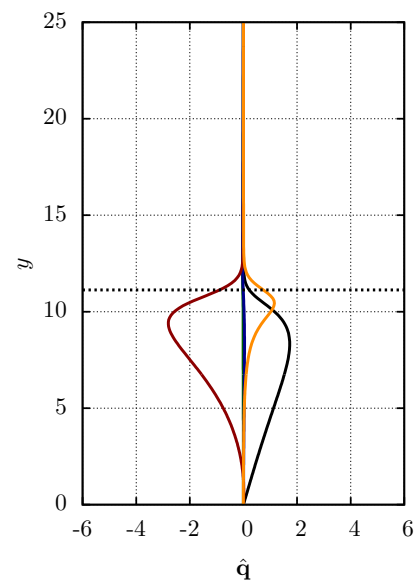

(c) $R / R_{1}=0.6$

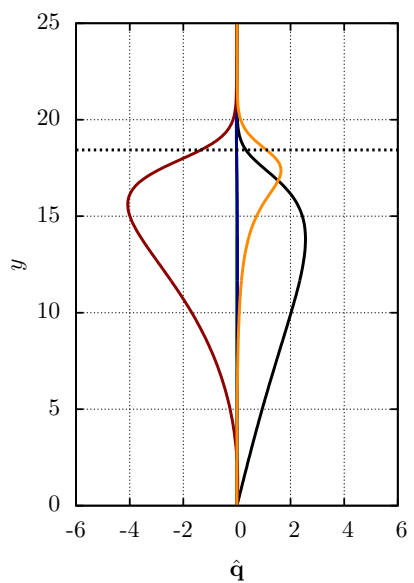

(d) $R / R_{1}=1.0$

Figure 7. Optimal disturbance vector at selected streamwise position for the Mach 6 boundary layer NS solution with $T_{w} / T_{a d}=0.8, R_{0} / R_{1}=0.1$ (i.e., $x_{0} / x_{1}=0.01$ ), $R_{1}=2,000$, and $\beta=0.116$

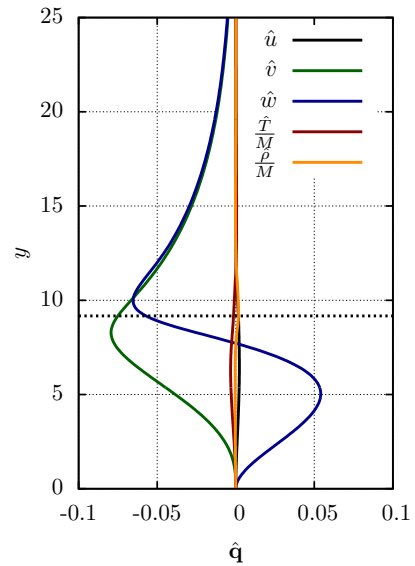

(a) $R / R_{1}=0.5$

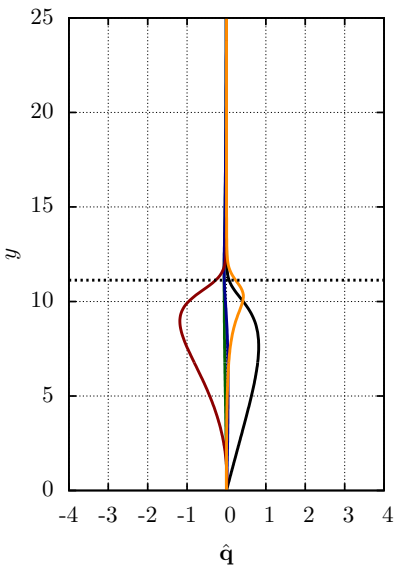

(b) $R / R_{1}=0.6$

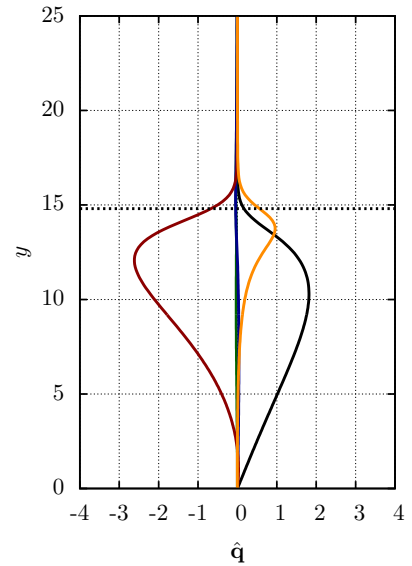

(c) $R / R_{1}=0.8$

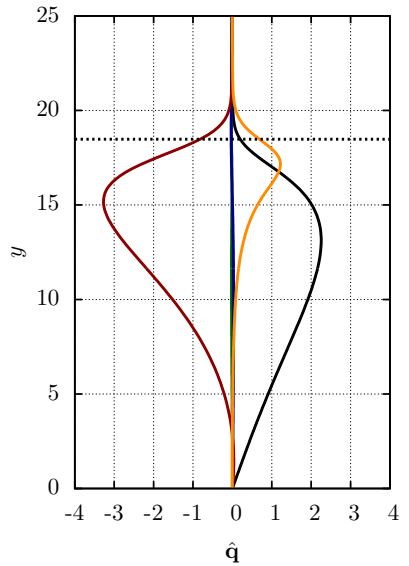

(d) $R / R_{1}=1.0$

Figure 8. Optimal disturbance vector at selected streamwise position for the Mach 6 boundary layer NS solution with $T_{w} / T_{a d}=0.8, R_{0} / R_{1}=0.5, R_{1}=1,000$, and $\beta=0.166$

Transient growth results for a Mach 10 boundary layer with $T_{w} / T_{a d}=0.35$ solution of the NS equations are shown in figures 9 (a) and 9 (b) for $R_{1}=1,000$ and $R_{1}=2,000$, respectively. The effect of $R_{0} / R_{1}$ is in line with the predictions at lower Mach numbers. Again, the maximum optimal energy gain is achieved for an initial location near $R_{0} / R_{1}=0.5$. Consistent with trends noted in the context of Mach 6 results, the effect of the shock layer is stronger at this higher Mach number. Thus, for initial disturbance locations close to the leading edge $\left(R_{0} / R_{1} \leq 0.3\right)$, the scaled optimal gain at $R_{1}=1,000$ (figure $9($ a) $)$ is significantly lower than that at $R_{1}=2,000$. Specifically, a reduction of $60 \%$ in the scaled optimal gain is observed with $R_{0} / R_{1}=0.2$ and $R_{1}=1,000$ and a reduction of $23 \%$ with the same $R_{0} / R_{1}$ and $R_{1}=2,000$, both with respect to the 
corresponding prediction based on the (shock-free) self-similar basic state. Also, at a fixed $R_{0} / R_{1}<0.4$, the presence of the leading-edge shock increases the optimum spanwise wavenumber for maximum growth. As observed in the Mach 6 cases, satisfactory results could not be obtained using the present numerical method for $R_{0}<200$ due to the strong viscous-inviscid interaction region and shock wave. The streamwise evolution of disturbance profiles is shown in figure 10 for $R_{1}=1,000, R_{0} / R_{1}=0.5$, and $\beta=0.124$. These profiles are similar in shape to those at Mach 6 with the same relative initial location $R_{0} / R_{1}$. Because of the lower surface temperature ratio in the present case $\left(T_{w} / T_{a d}=0.35\right)$ vis a vis that in figure 8 for the Mach 6 case $\left(T_{w} / T_{a d}=0.8\right)$, the temperature perturbation has a stronger positive (local) maximum near the surface. The presence of this local maximum for $T_{w} / T_{a d}<1$ was also noted in the $M=3$ case by Tumin \& Reshotko. ${ }^{17}$

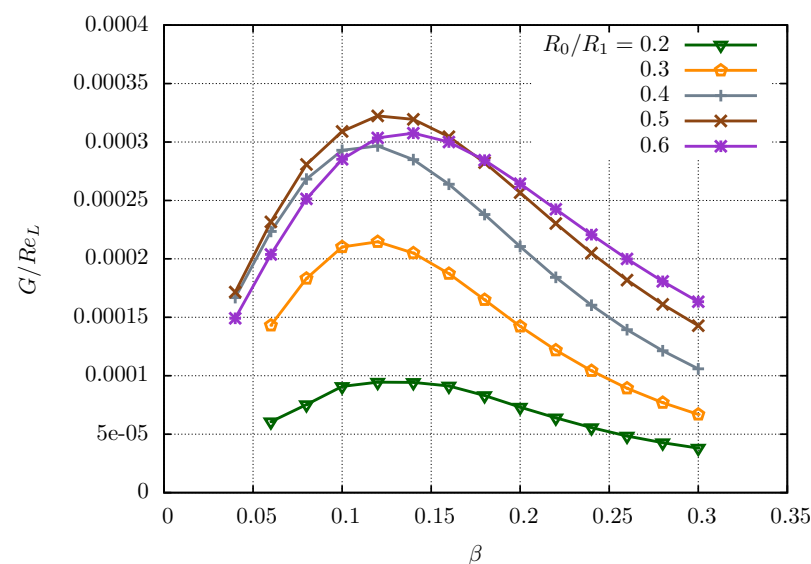

(a) $R_{1}=1,000$

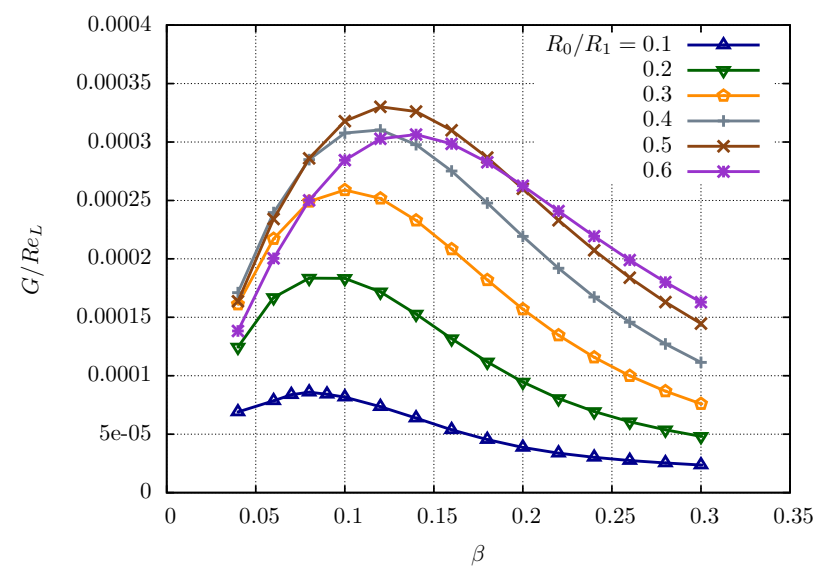

(b) $R_{1}=2,000$

Figure 9. Effect of initial position $R_{0}$ on optimal energy gain at (a) $R_{1}=1,000$ and (b) $R_{1}=2,000$. Basic state corresponds to NS solution at $M=10$ and $T_{w} / T_{a d}=0.35$.

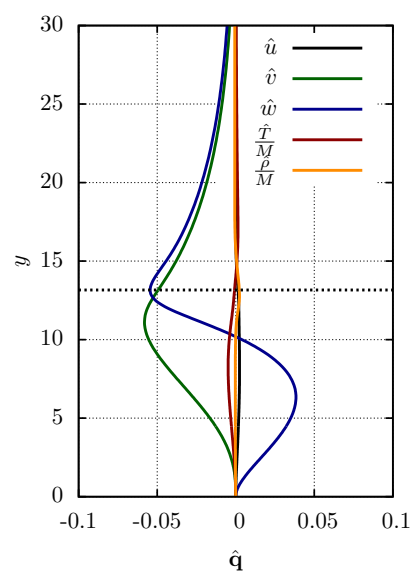

(a) $R / R_{1}=0.5$

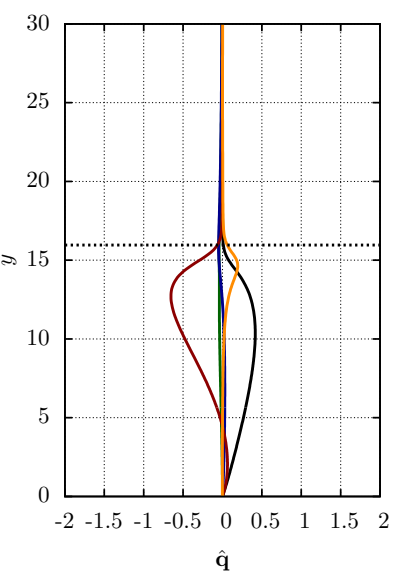

(b) $R / R_{1}=0.6$

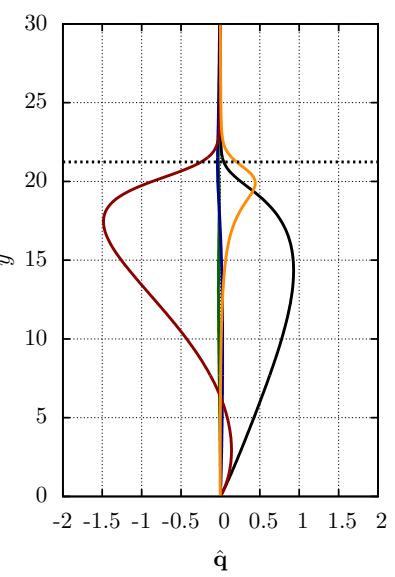

(c) $R / R_{1}=0.8$

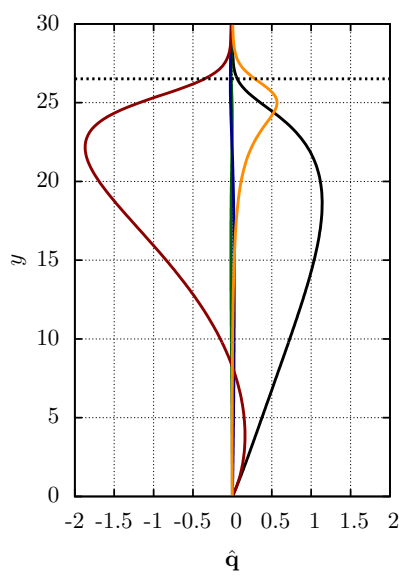

(d) $R / R_{1}=1.0$

Figure 10. Optimal disturbance vector at selected streamwise position for the Mach 6 boundary layer NS solution with $T_{w} / T_{a d}=0.35, R_{0} / R_{1}=0.5, R_{1}=1,000$, and $\beta=0.126$.

\section{III.C. Effects of Wall-Cooling and Mach Number on Transient Growth}

The effects of wall-cooling and Mach numbers on transient growth are further studied using both self-similar solutions to boundary layer equations form $M=0$ to $M=10$ and the previously analyzed NS basic states. The transient growth results, i.e., optimum energy gain and corresponding spanwise wavenumber, are found to correlate better when the boundary layer thickness, $\delta_{.995}$, is used as the reference length scale rather than the similarity scale $\delta$. The rescaled energy gain is defined as $\sqrt{G} / R e_{.995}$ and the rescaled spanwise wavenumber is defined as $\beta_{\delta .995}=\beta \delta_{.995}$. 
Figure 11 shows the rescaled optimum energy gains and corresponding rescaled spanwise wavenumbers for boundary layer flows with $T_{w} / T_{a d}=1$ and $M \leq 3$ for an initial disturbance location near the leading edge. Boundary layers at higher Mach numbers are not included in this figure because of previously mentioned convergence difficulties due to the presence of the basic state shock very close to the boundary layer edge. Following the rescaling by $\delta_{.995}$, the maximum optimal gain increases with $M$ and the corresponding spanwise wavenumber slightly decreases over the selected range of Mach numbers. The optimum value of the spanwise wavenumber falls within the narrow range of $\beta_{\delta_{.995}} \in[2.3,2.4]$.

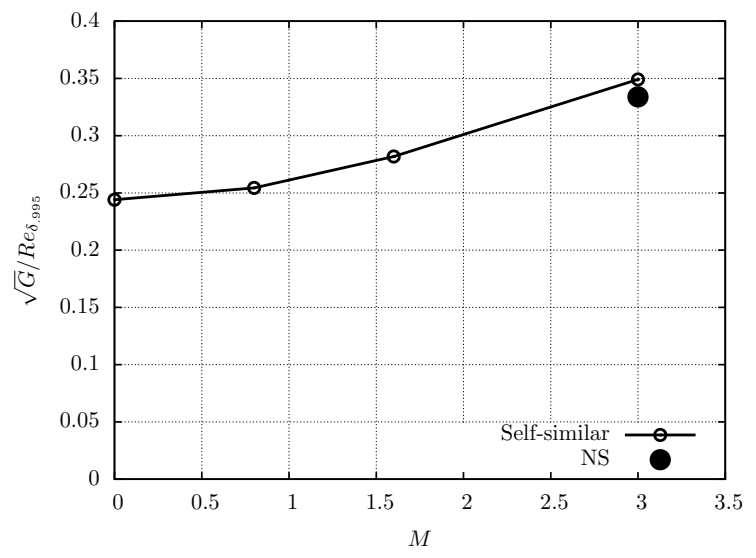

(a) Rescaled energy gain

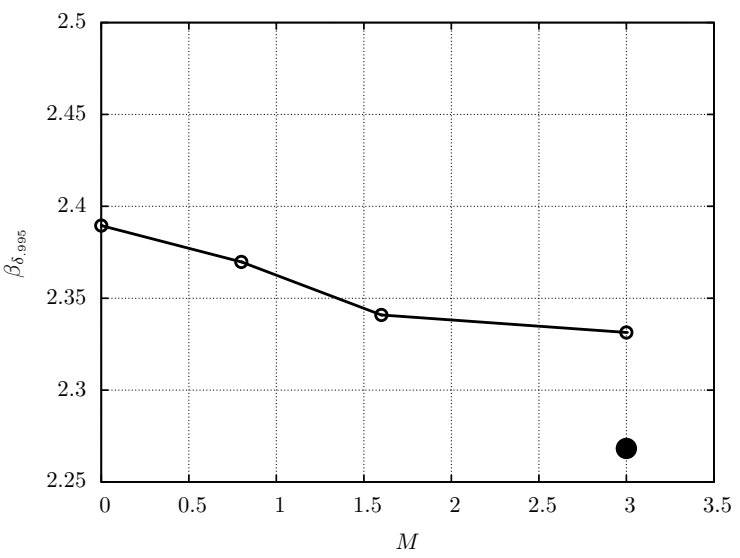

(b) Rescaled spanwise wavenumber (same legend as (a))

Figure 11. (a) Rescaled optimum energy gains and (b) corresponding rescaled spanwise wavenumbers with initial optimization position at the leading edge, $x_{0}=0$, for adiabatic wall and selected Mach numbers. Open symbols with solid line denote results obtained with self-similar basic states and the filled symbol at Mach 3 represents the result obtained with a NS basic state with $R_{1}=1000$.

Figure 12 shows a the outcome of a similar parametric study for Mach numbers up to 10 and various values of $T_{w} / T_{a d}$ with the initial position corresponding to $R_{0} / R_{1}=0.5$, which approximates the initial position for maximum transient growth (Subsection III.B). As observed in figure 12(a), the rescaled maximum optimal gain increases approximately linearly with the wall temperature ratio, $T_{w} / T_{a d}$. Also, $\sqrt{G} / R e_{\delta .995}$ increases with the Mach number for constant $T_{w} / T_{a d}$. The limited set of results obtained using the NS basic states agree with the self-similar results. The small reduction produced by the shock layer is hardly visible on the scale of this plot. Figure 12(b) shows that the rescaled wavenumber also increases with increasing $T_{w} / T_{a d}$ and $M$, and the rate of increase with $T_{w} / T_{a d}$ becomes greater at larger $M$. Thus, $\beta_{\delta .995}$ increases very slowly with $T_{w} / T_{a d}$ at $M=3$, but the rise becomes significantly more rapid at $M=10$. In this figure, the effect of the leading edge shock wave is small but noticeable, indicating a higher spanwise wavenumber relative to prediction based on the self-similar mean flow.

The total disturbance energy used to characterize the energy gain defined by equation (4) includes the kinetic energy component and a component involving contributions from fluctuations in thermodynamic fluctuations. To characterize the relative variation in these components as a function of flow conditions, figure 12(c) plots the ratio of kinetic energy and total energy at the final station. Because the secondary instability of transient growth disturbances is mostly driven by streamwise velocity shear, ${ }^{49,50}$ a greater value of this ratio is likely to enhance the growth of shear layer instabilities and possibly result in an earlier onset of bypass transition associated with a nonlinear disturbance. At $M=10^{-3}$, nearly all disturbance energy corresponds to the kinetic energy. As the Mach number increases to supersonic and hypersonic values, the contribution of kinetic energy decreases progressively. At $M=10$, less than one half of the total energy comes from kinetic energy, regardless of the surface temperature condition. The relative energy contribution from thermodynamic fluctuations increases with $T_{w} / T_{a d}$. In other words, the kinetic energy component gains in significance as the surface is cooled, which would likely destabilize the secondary instabilities.

\section{Pressure Gradient Effect: Transient Growth in Planar Stagnation Point Boundary Layer}

Transient growth in a boundary layer flow developing under a favorable pressure gradient is examined in this subsection. Specifically, the basic state corresponds to a planar stagnation point boundary layer, 


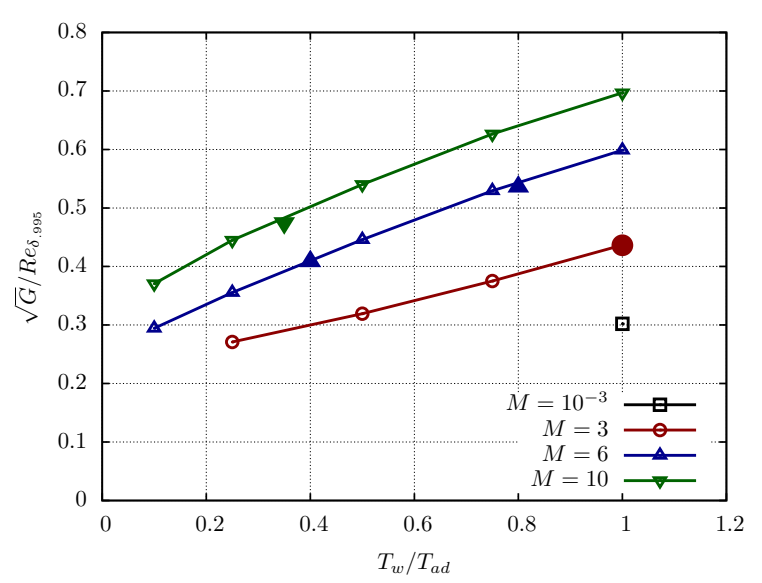

(a) Rescaled energy gain

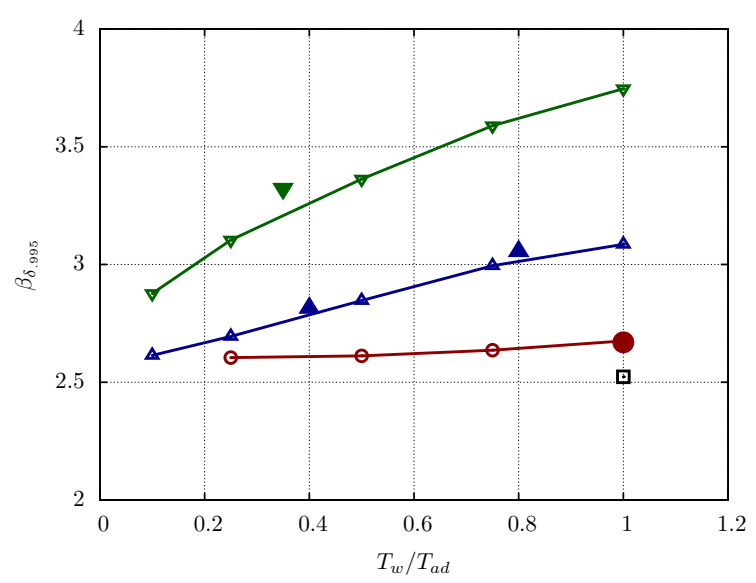

(b) Rescaled spanwise wavenumber (same legend as (a))

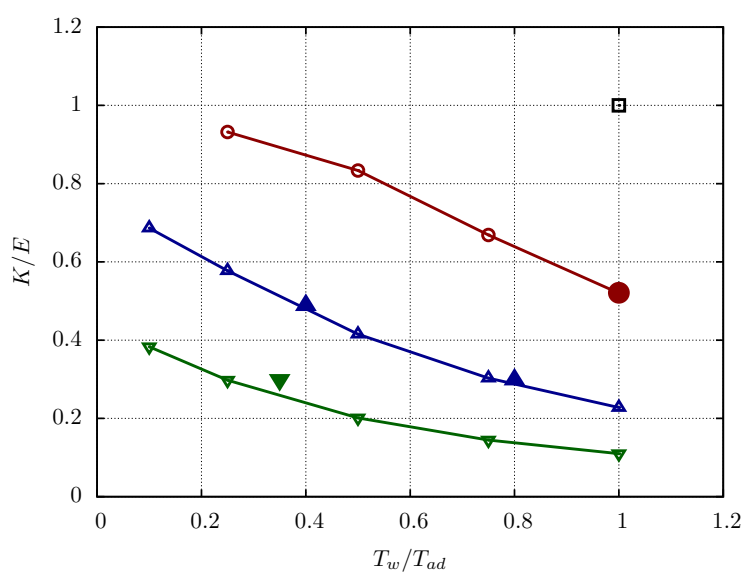

(c) Ratio between kinetic energy and total energy at $R_{1}$ (same legend as (a))

Figure 12. (a) Rescaled optimum energy gains, (b) corresponding rescaled spanwise wavenumbers, and (c) ratio of kinetic energy and total energy of the optimal disturbance at the final station with $R_{0} / R_{1}=0.5$ and $R_{1}=1,000$ for selected Mach numbers and $T_{w} / T_{a d}$. Open symbols with solid line denote results obtained with self-similar basic states and the filled symbols represent the result obtained with NS basic states. 
which is described by the exact solution found by Hiemenz ${ }^{51,52}$ to the NS equations. This boundary layer flow is known to be linearly stable at all streamwise locations (or, equivalently, for all Reynolds numbers). Hence, any linear disturbance growth must occur via the transient growth mechanism. For the Hiemenz flow, the boundary layer thickness remains constant at all stations; and the edge velocity increases linearly with distance $x^{*}$ from the stagnation location, i.e., $u_{\infty}^{*}=c x^{*}$. The wall-normal length scale is defined as $l_{r}^{*}=\sqrt{\nu^{*} / c}$, where $c$ is the inviscid strain rate near the stagnation point, i.e., $c=d u_{\infty}^{*} / d x^{*}$. The local Reynolds number $R$ is defined as $R=u_{\infty}^{*} l_{r}^{*} / \nu^{*}$, which leads to $R=x$. Due to the lack of a velocity scale in this case, the optimal gain scales linearly with $R_{1}^{2}$; and hence, the optimal growth behavior of the Hiemenz flow is characterized by the single parameter $x_{0} / x_{1}$, which is also the same as $R_{0} / R_{1}$ because of the constant boundary layer thickness.

The effect of $x_{0} / x_{1}$ on the optimal gain factor as a function of the spanwise wavenumber is shown in figure 13(a), where the ordinate is scaled by $R_{1}^{2}$ as mentioned above. The sensitivity of the results to $R_{1}$ was checked using $R_{1}=1,000$ and $R_{1}=10,000$, and no visible differences were found. Results are plotted for inflow locations ranging from very close to the stagnation point to just upstream of the location of interest, $x_{1}$. Although not shown, for inflow locations just upstream of the target location, the transient growth magnitude is very small. As the inflow location $x_{0}$ moves progressively farther upstream of $x_{1}$, i.e., as the length of transient growth region increases, the optimal gain factor continues to increase until reaching a local maximum near $x_{0} / x_{1} \approx 0.84$. The corresponding nondimensional wavenumber is $\beta=1.13$. The optimal gain factor decreases beyond this peak, reaching a minimum when the inflow location corresponds to $x_{0} / x_{1} \approx 0.3$ (and the corresponding spanwise wavenumber is $\beta \approx 0.60$ ). As the inflow location moves closer to the stagnation point, the optimal gain continues to increase in a monotonic fashion, at least until $x_{0} / x_{1}=0.02$ (i.e., the smallest value of $x_{0} / x_{1}$ for which calculations were performed). The optimal wavenumber for inflow locations near the stagnation point is significantly smaller than the values noted above in the context of the local peak for $x_{0} / x_{1} \approx 0.84$ and the minimum energy gain at $x_{0} / x_{1} \approx 0.3$.

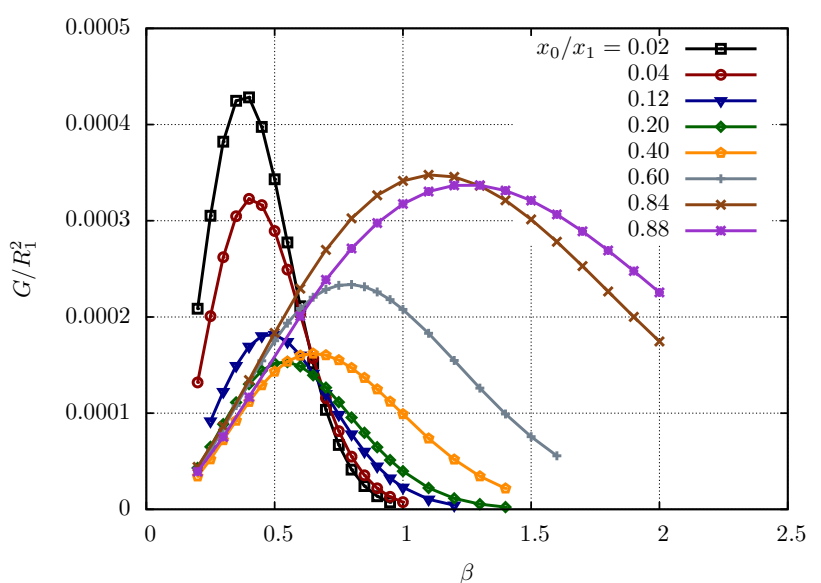

(a) Optimal energy gain

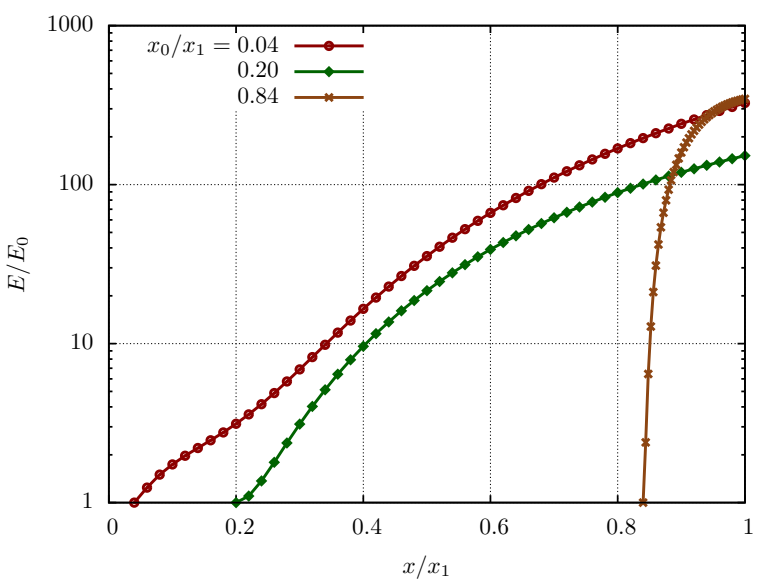

(b) Energy evolution

Figure 13. (a) Effect of initial optimization position, $x_{0}$, in the optimal energy gain for the incompressible planar Hiemenz boundary layer flow. (b) Energy evolution with $x_{1}=1,000$ for $x_{0} / x_{1}=0.04(\beta=0.41), x_{0} / x_{1}=0.2(\beta=0.54)$, and $x_{0} / x_{1}=0.84(\beta=1.13)$.

The streamwise evolution of the optimal gain factor for three selected inflow locations is plotted in figure 13(b). The gain factor for $x_{0} / x_{1} \approx 0.84$ is nearly the same as that for $x_{0} / x_{1} \approx 0.04$, but this growth occurs over a considerably smaller distance. Therefore, the average rate of increase in disturbance energy norm is nearly six times higher for the inflow location corresponding to $x_{0} / x_{1} \approx 0.84$.

The evolution of disturbance shape functions for inflow locations at $x_{0} / x_{1}=0.04,0.2$, and 0.84 is shown in figures 14 to 16, respectively. Part (a) in each figure illustrates the optimal initial profiles for the respective inflow location. Regardless of the inflow location, the optimal initial profiles correspond to a streamwise vortex; and the disturbance progressively evolves into a streamwise streak dominated by the streamwise velocity perturbation, exactly similar to the disturbance evolution over the flat plate configurations as noted in Section III. However, whereas the wall-normal support of the disturbance shape functions at the initial and final streamwise locations was comparable to each other in the flat plate cases, the shape functions in the stagnation point case exhibit increasingly slower decay along the wall-normal direction for streamwise 
locations near the inflow station. For instance, for $x_{0} / x_{1}=0.04$, the peak of the $\hat{v}$ component of velocity occurs at $y \approx 35$, which is more than an order of magnitude larger than the thickness of the boundary layer. Even at $y \approx 100$, the $\hat{v}$-velocity perturbation has decayed by a factor of at most 2 relative to the maximum of the disturbance shape function. Furthermore, the dominant component of the inflow disturbance shape corresponds to the $\hat{v}$ (i.e., wall normal) velocity component, which corresponds to the direction of incoming flow approaching the stagnation point. The disparity between the wall-normal extents of the disturbance shape functions at the inflow and optimization locations becomes increasingly weaker as the inflow location moves closer to the optimization location. For the case of $x_{0} / x_{1}=0.84$, for instance, the wall-normal support of the inflow shape functions is at most twice as large as that near the outflow location $x_{1}$.

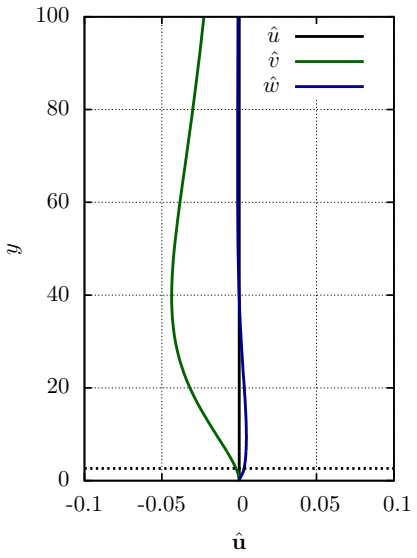

(a) $x / x_{1}=0.04$

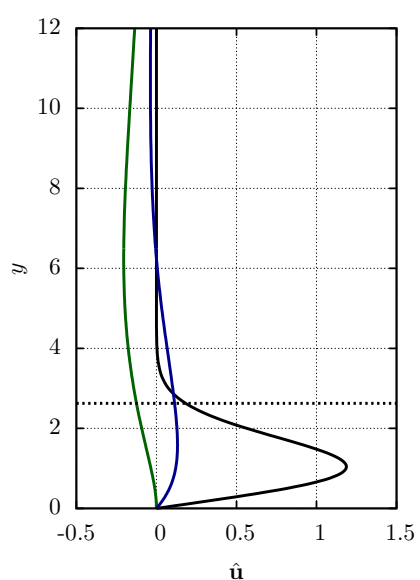

(d) $x / x_{1}=0.4$

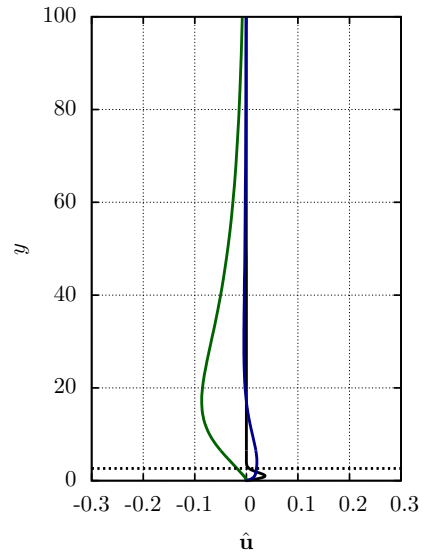

(b) $x / x_{1}=0.1$

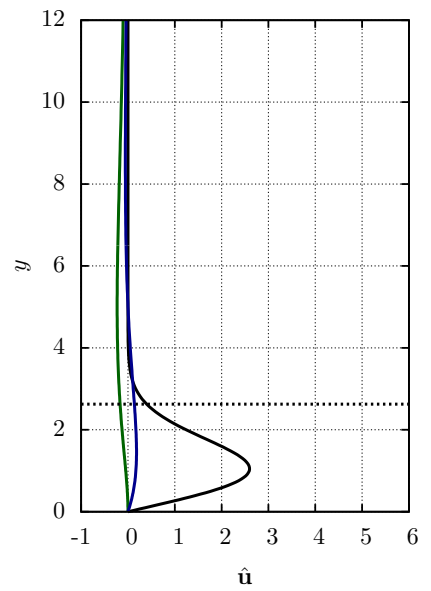

(e) $x / x_{1}=0.6$

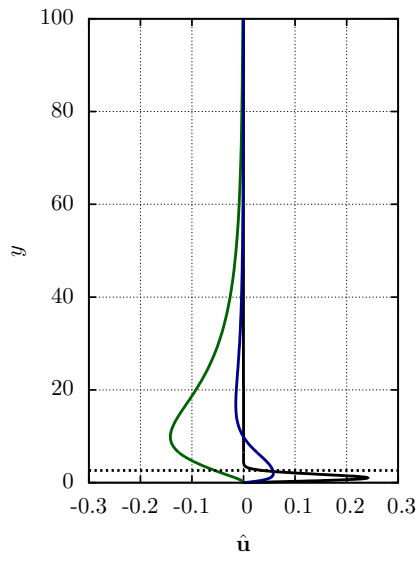

(c) $x / x_{1}=0.2$

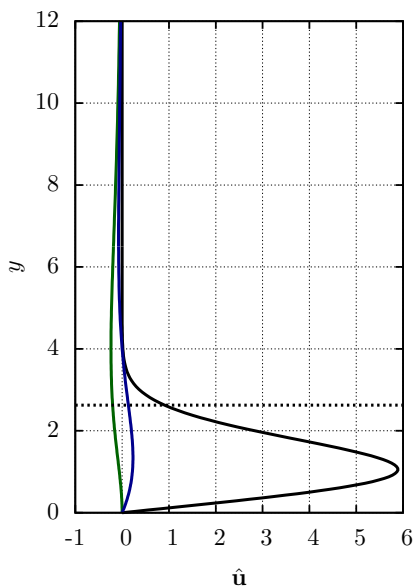

(f) $x / x_{1}=1.0$

Figure 14. Optimal disturbance vector at selected streamwise positions for the incompressible planar Hiemenz boundary layer flow with $R_{1}=1,000, x_{0} / x_{1}=0.04$ and $\beta=0.41$.

The transient growth results for the stagnation point boundary layer present an interesting contrast to the flat plate results in previous subsections. Figure 17 shows a comparison of the respective values of rescaled optimal gain and the associated spanwise wavenumbers as a function of the initial optimization position. The rescaled optimal energy gains and spanwise wavenumbers are greater for the Blasius flow at every selected $R_{0} / R_{1}$. The overall maximum of the rescaled energy gin for the Blasius flow occurs at $R_{0} / R_{1}=\sqrt{x_{0} / x_{1}}=0.6$, where $\sqrt{G} / R_{\delta_{.995}}=0.309$ and $\beta_{\delta_{.995}}=2.79$. For the Hiemenz flow, the overall maximum is found at $R_{0} / R_{1}=x_{0} / x_{1}=0.84$ with $\sqrt{G} / R_{\delta_{.995}}=0.0489$ and $\beta_{\delta_{.995}}=2.96$. Not surprisingly, however, these results are significantly lower than those for the Blasius boundary layer, but rather similar to those of Refs. 18 and 19 for azimuthally periodic disturbances in an axisymmetric stagnation point flow. The latter case includes the additional complexity of a decreasing disturbance wavelength in the azimuthal direction as the inflow location approaches the stagnation point. The similarity of transient growth characteristics in that case and the planar stagnation point boundary layer considered herein suggests that the 


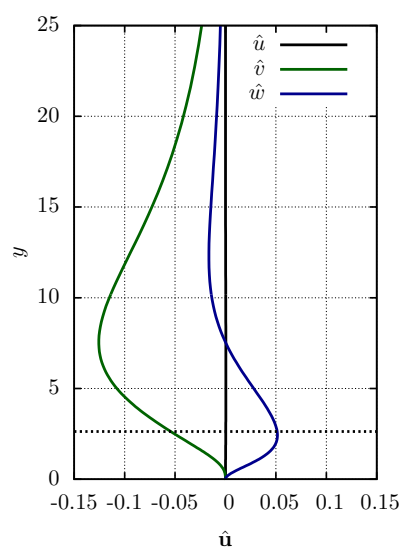

(a) $x / x_{1}=0.2$

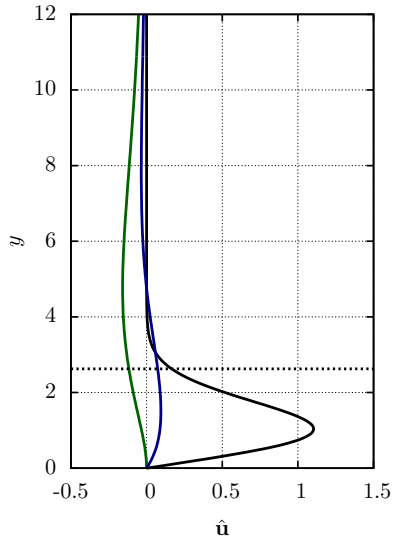

(b) $x / x_{1}=0.4$

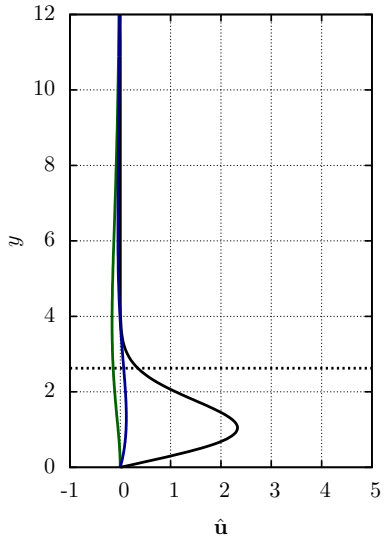

(c) $x / x_{1}=0.6$

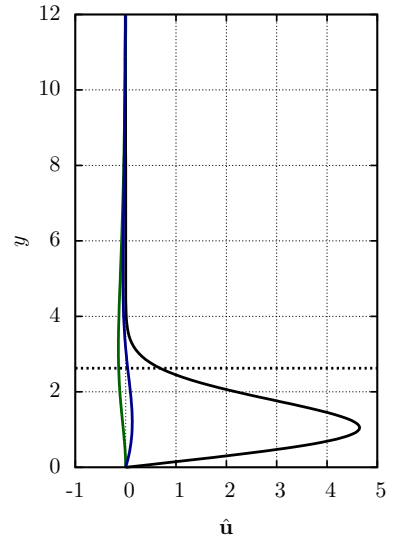

(d) $x / x_{1}=1.0$

Figure 15. Optimal disturbance vector at selected streamwise positions for the incompressible planar Hiemenz boundary layer flow with $R_{1}=1,000, x_{0} / x_{1}=0.2$ and $\beta=0.54$.

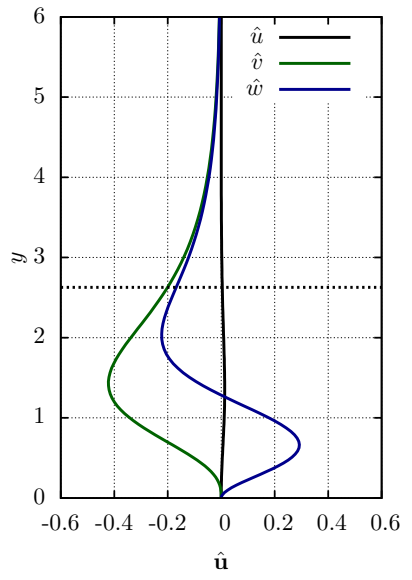

(a) $x / x_{1}=0.84$

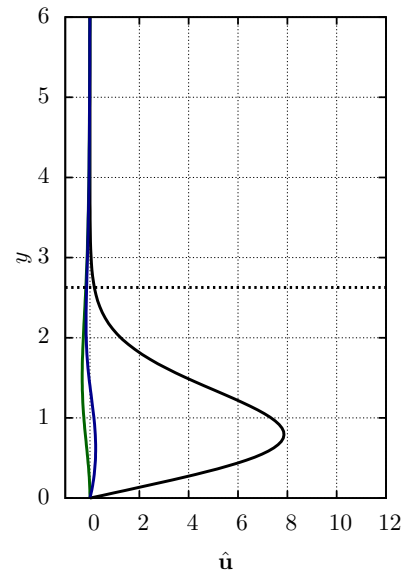

(b) $x / x_{1}=0.90$

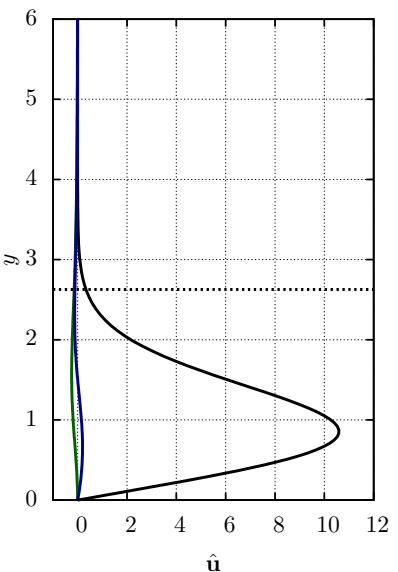

(c) $x / x_{1}=0.96$

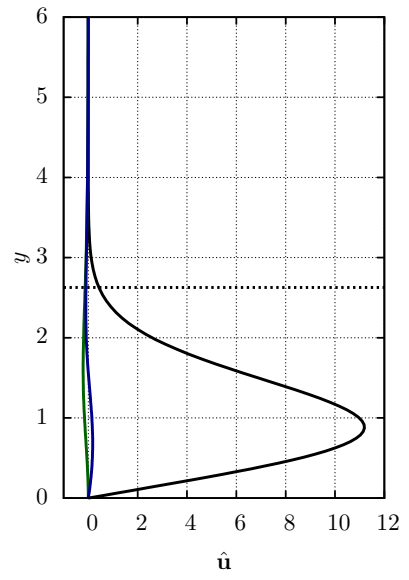

(d) $x / x_{1}=1.0$

Figure 16. Optimal disturbance vector at selected streamwise positions for the incompressible planar Hiemenz boundary layer flow with $R_{1}=1,000, x_{0} / x_{1}=0.84$ and $\beta=1.13$. 
flow acceleration is the dominant effect in comparison with the effect of a collapsing azimuthal length scale. Even though the basic state for the planar stagnation point represents an exact solution to the NS equations in this case, the PSE represent an approximate set of governing equations for the disturbance field. Hence, direct numerical simulations of transient growth are desired to evaluate the accuracy of the PSE predictions presented herein. The increasing "protrusion" of the disturbance shape functions into the freestream region has potential implications for the excitation of the transient growth disturbances in a realistic freestream disturbance environment and those implications should be assessed in future work as well. On the other hand, disturbances at the body surface such as wall roughness are unlikely to induce disturbance profiles that extend as far out as the optimal initial conditions near the stagnation point. Therefore, the growth of stationary disturbances induced by surface roughness in the vicinity of the stagnation point is likely to be suboptimal. Additional computations based on initial disturbance profiles that are primarily confined to the boundary layer region would help determine just how suboptimal the associated transient growth is likely to be.

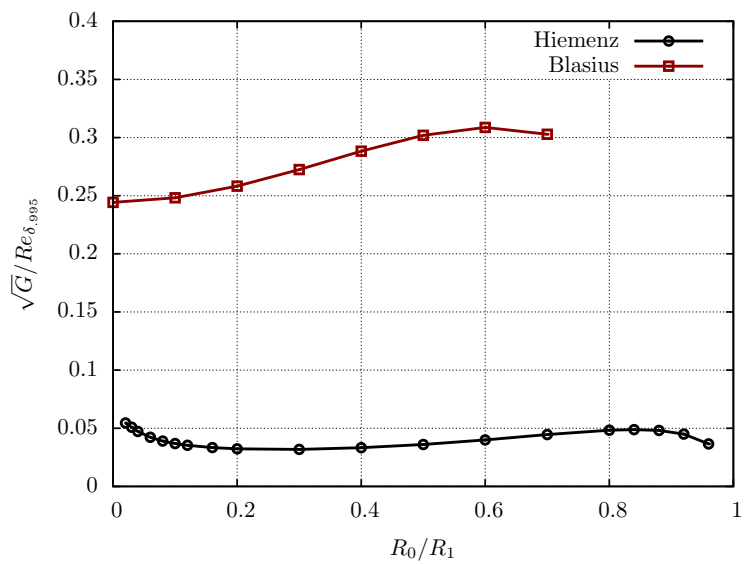

(a) Rescaled optimum gain

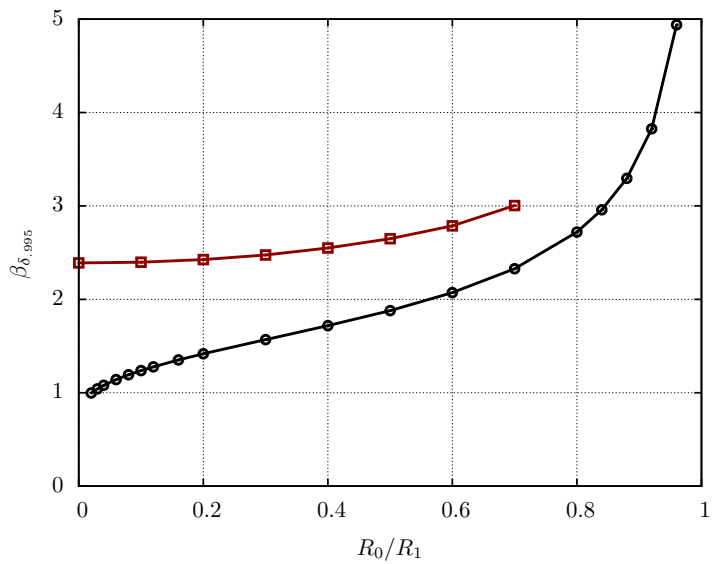

(b) Rescaled spanwise wavenumber

Figure 17. Comparison between (a) rescaled optimum energy gains and (b) corresponding rescaled spanwise wavenumbers for the Hiemenz and Blasius boundary layer flows

\section{Summary and Conclusions}

Optimal transient growth in compressible, zero-pressure-gradient boundary layer flows has been studied using a variational approach based on the parabolized stability equations (PSE). The effects of viscousinviscid interaction near the leading edge and the weak shock wave are addressed using mean flows based on the NS equations. Optimal growth results are shown for compressible flat plate boundary layer flow solutions of the NS equations for four cases, namely, $M=3\left(T_{w} / T_{a d}=1.0\right), M=6\left(T_{w} / T_{a d}=0.4\right.$ and 0.8$)$, and $M=10\left(T_{w} / T_{a d}=0.35\right)$. At Mach 3, when the initial optimization position is located near the leading edge, the difference between energy gains based on the NS mean flow and the self-similar, boundary layer approximation is rather small, less than approximately 9\%. At higher Mach number, this effect becomes relevant. For the Mach $10\left(T_{w} / T_{a d}=0.35\right)$ case, the scaled optimal gain with initial disturbance location at $R_{0} / R_{1}=0.2$ is reduced with respect to predictions based on the (shock-free) self-similar mean flow by $60 \%$ with the final optimization position at $R_{1}=1000$ and by $23 \%$ when $R_{1}=2000$. For the higher Mach number cases, the stronger inviscid-viscous interaction and shock wave in the NS mean flow prevents the solution of converged results for initial optimization positions near the leading edge. For the downstream initial locations that yielded satisfactory optimal growth predictions, the main effect of the shock wave on the transient growth results was a small reduction in the optimum energy gain and small increase of the corresponding spanwise wavenumber. Calculations for various initial locations show that the highest optimal growth occurs for an initial location of $x_{0} / x_{1} \approx 0.25\left(R_{0} / R_{1} \approx 0.5\right)$, where the effects of the leading edge shock are relatively weak for the optimization station of interest $\left(R_{1}=1000\right)$. Hence, the difference between optimal gain predictions based on the self-similar approximation and the NS solution is less than $6 \%$ of relative error for the selected cases. For $x_{0} / x_{1}=0.25$, the reduction in optimal growth based on the NS mean flow solution increases to approximately $3.4 \%$ at Mach 6 with $T_{w} / T_{a d}=0.8$ and to $6.1 \%$ at Mach 10 
with $T_{w} / T_{a d}=0.35$.

The effect of Mach number and wall-cooling is studied by using self-similar and NS basic states. Setting the leading-edge as initial optimization position for boundary layer flows with $T_{w} / T_{a d}=1$ and $M \leq 3$, the optimum nondimensional wavenumber scaled with respect to $\delta .995$ is found to lie within the narrow range of $(2.3,2.4)$ for $M \leq 3$ with $T_{w} / T_{a d}=1$. The rescaled energy gain, $\sqrt{G} / \operatorname{Re}_{\delta .995}$, shows a monotonic increase with increasing Mach numbers. When the initial location for transient growth is set to $x_{0} / x_{1}=0.25$ $\left(R_{0} / R_{1}=0.5\right)$, which approximately corresponds to the maximum energy gain over all possible inflow locations, the rescaled optimal wavenumber is found to increase with the flow Mach number over $M \in[0,10]$, and also with the wall temperature ratio $T_{w} / T_{a d}$. The same trend is observed for the rescaled energy gain. The ratio of kinetic energy to total energy of the disturbance, $K / E$, is studied for the optimal spanwise wavenumbers for a variety of freestream conditions. Because the secondary instability of transient growth disturbances is mostly driven by streamwise velocity shear, ${ }^{49,50}$ a greater value of this ratio is likely to enhance the growth of shear layer instabilities and possibly result in an earlier onset of bypass transition associated with a nonlinear disturbance. As the Mach number increases, $K / E$ is found to decrease with Mach number and increase with $T_{w} / T_{a d}$. Therefore, the wall-cooling effect is likely to enhance the consequences of transient growth in two different ways: it increases both the maximum energy gain and the percentage of disturbance kinetic energy.

The effect of pressure gradient on transient growth results is addressed in a canonical setting by analyzing the incompressible planar stagnation flow. The boundary layer solution is given by the Hiemenz flow. Again, the optimal initial perturbation corresponds to a streamwise vortex that evolves into a streamwise streak, as in the zero-pressure-gradient flate plate cases. However, in comparison with the Blasius boundary layer, the rescaled optimum gain and spanwise wavenumbers are found to be lower in the presence of the favorable pressure gradient. Furthermore, there is a dramatic change in the dependence of optimal energy gain on the initial location. In addition to a maximum at $R_{0} / R_{1}=0.84$, one observes a local minimum at $R_{0} / R_{1}=0.3$ in the optimum gain as a function of the initial location. For $R_{0} / R_{1}<0.3$, the optimal gain increases with decreasing $R_{0}$. Looking at the optimal initial disturbance profiles for initial positions close to the leading edge, e.g., $x_{0} / x_{1}=0.04$, the wall-normal extension of the wall normal velocity is found to be several orders of magnitude larger than the constant boundary layer thickness of the Hiemenz flow. Because optimal initial perturbations of this type are unlikely to be generated by surface disturbances, energy gain due to roughness induced transient growth near the stagnation point is likely to be significantly suboptimal. Of course, the actual disturbance amplitudes would be determined by the combined effects of the favorable pressure gradient on the (reduced) optimal gain and the realizability of the optimal initial disturbance in a typical disturbance environment. The effect of pressure gradient on realizable transient growth is suggested as a topic for future research.

\section{Acknowledgments}

This work is supported by NASA's Transformational Tools \& Technologies project. The authors would like to thank Prof. Ardeshir Hanifi from the Royal Institute of Technology (KTH) in Stockholm, Sweden, for his gracious assistance during the initial phase of this work.

\section{References}

\footnotetext{
${ }^{1}$ Mack, L. M., "Boundary layer linear stability theory," AGARD-R-709 Special course on stability and transition of laminar flow, 1984, pp. 3.1-3.81.

${ }^{2}$ Schmid, P. and Henningson, D. S., Stability and Transition in Shear Flows, Springer, New York, 2001.

${ }^{3}$ Saric, W. and Nayfeh, A., "Nonparallel stability of boundary-layer flow," Phys. Fluids, Vol. 18, No. 10, 1975, pp. 945-950.

${ }^{4}$ Herbert, T., "Parabolized stability equations," Ann. Rev. Fluid Mech., Vol. 29, 1997, pp. 245-283.

${ }^{5}$ Landahl, M., "A note on algebraic instability of inviscid parallel shear flows," J. Fluid Mech., Vol. 98, 1980, pp. $243-251$.

${ }^{6}$ Ellingsen, T. and Palm, E., "Stability of linear flow," Phys. Fluids, Vol. 18, No. 4, 1975, pp. 487-488.

${ }^{7}$ Butler, K. and Farrell, B., "Three-dimensional optimal perturbations in viscous shear flow," Physics of Fluids, Vol. 4, No. 8, 1992, pp. 1637-1650.

${ }^{8}$ Reddy, S. and Henningson, D., "Energy growth in viscous channel flow," J. Fluid Mech., Vol. 252, 1993, pp. 209-238.

${ }^{9}$ Trefethen, L. N., Trefethen, A. E., Reddy, S. C., and Driscoll, T., "Hydrodynamic stability without eigenvalues," Science, Vol. 261, 1993, pp. 578-584.

${ }^{10}$ Hanifi, A., Schmid, P., and Henningson, D., "Transient growth in compressible boundary layer flow," Phys. Fluids, Vol. 8, 1996, pp. 51-65.
} 
${ }^{11}$ Andersson, P., Berggren, M., and Henningson, D., "Optimal disturbances and bypass transition in boundary layers," Phys. Fluids, Vol. 11, 1999, pp. 134-150.

${ }^{12}$ Luchini, P., "Reynolds-number-independent instability of the boundary layer over a flat surface: Optimal perturbations," J. Fluid Mech., Vol. 404, 2000, pp. 289-309.

${ }^{13}$ Kemp, N., The laminar three-dimensional boundary layer and a study of the flow past a side edge, Master's thesis, Cornell University, 1951.

${ }^{14}$ Davis, R. and Rubin, S., "Non-Navier Stokes viscous flow computations," Compt. Fluids, Vol. 8, 1980, pp. $101-131$.

${ }^{15}$ Goldstein, M., Sescu, A., Duck, P., and Choudhari, M., "Algebraic/transcendental disturbance growth behind a row of roughness elements," J. Fluid Mech., Vol. 668, 2011, pp. 236-266.

${ }^{16}$ Tumin, A. and Reshotko, E., "Spatial theory of optimal disturbances in boundary layers," Phys. Fluids, Vol. 13, 2001, pp. 2097-2104.

${ }^{17}$ Tumin, A. and Reshotko, E., "Optimal disturbances in compressible boundary layers," AIAA J., Vol. 41, 2003, pp. 23572363.

${ }^{18}$ Tumin, A. and Reshotko, E., "Optimal disturbances in the boundary layer over a sphere," AIAA Paper 2004-2241, 2004.

${ }^{19}$ Zuccher, S., Tumin, A., and Reshotko, E., "Parabolic approach to optimal perturbations in compressible boundary layers," J. Fluid Mech., Vol. 556, 2006, pp. 189-216.

${ }^{20}$ Zuccher, S., Shalaev, I., Tumin, A., and Reshotko, E., "Optimal Disturbances in the supersonic boundary layer past a sharp cone," AIAA J., Vol. 45, 2007, pp. 366-373.

${ }^{21}$ Reshotko, E., "Transient growth: A factor in bypass transition," Phys. Fluids, Vol. 13, 2001, pp. 1067-1075.

${ }^{22}$ Morkovin, M., Reshotko, E., and Herbert, T., "Transition in open flow systems - A reassessment," Bull. Am. Phys. Soc., Vol. 39, 1994, pp. 1882.

${ }^{23}$ Reshotko, E., "Boundary layer transition, instability and control," AIAA Paper 94-0001, 1994.

${ }^{24}$ Reshotko, E. and Tumin, A., "Spatial theory of optimal disturbances in a circular pipe flow," Phys. Fluids, Vol. 13, 2001, pp. 991-996.

${ }^{25}$ Reshotko, E., "Preliminary experimental study of disturbances in a laminar boundary layer due to distributed surface roughness," AIAA Paper 81-1224, 1981.

${ }^{26}$ White, E., "Transient growth of stationary disturbances in a flat plate boundary layer," Phys. Fluids, Vol. 14, No. 12, 2002, pp. 4429-4439.

${ }^{27}$ Sharp, N. and White, E., "Roughness-induced transient growth on a hypersonic blunt cone," AIAA Paper 2014-0432, 2014.

${ }^{28}$ Murphy, J. and Rubesin, M., "Re-evaluation of heat-transfer data obtained in flight test of heat-sink shielded re-entry vehicles," J. Spacecraft, Vol. 3, No. 1, 1966, pp. 53-60.

${ }^{29}$ Reshotko, E. and Tumin, A., "The blunt body paradox - A case for transient growth," Proc. of the IUTAM LaminarTurbulent Symposium V, edited by H. Fasel and W. Saric, Sedona, AZ, USA, 2000, pp. 403-408.

${ }^{30}$ Schneider, S., "Hypersonic boundary-layer transition on blunt bodies with roughness," AIAA Paper 2008-0501, 2008.

${ }^{31}$ Theiss, A., Hein, S., Heitmann, D., Ali, S., and Radespiel, R., "Numerical and experimental investigation of laminarturbulent boundary layer transition on a blunt generic re-entry capsule," AIAA Paper 2014-2353, 2014.

${ }^{32}$ Choudhari, M. and Fischer, P., "Roughness induced transient growth," AIAA Paper 2005-4765, 2005.

${ }^{33}$ Leib, S., Wundrow, D., and Goldstein, M., "Effect of free-stream turbulence and other vortical disturbances on a laminar boundary layer," J. Fluid Mech., Vol. 380, 1999, pp. 169-203.

${ }^{34}$ Pralits, J., Airiau, C., Hanifi, A., Henningson, D., and Luchini, P., "Optimal disturbances in three-dimensional boundarylayer flows," Ercoftac Bull., Vol. 74, 2007, pp. 23-31.

${ }^{35}$ Tempelmann, D., Hanifi, A., and Henningson, D., "Spatial optimal growth in three-dimensoinal boundary layers," $J$. Fluid Mech., Vol. 646, 2010, pp. 5-37.

${ }^{36}$ Tempelmann, D., Hanifi, A., and Henningson, D., "Spatial optimal growth in three-dimensoinal compressible boundary layers," J. Fluid Mech., Vol. 704, 2012, pp. 251-279.

${ }^{37}$ Levin, O. and Henningson, D., "Exponential vs algebraic growth and transition prediction in boundary layer flow," Flow Turbul. Combust., Vol. 70, 2003, pp. 183-210.

${ }^{38}$ Pralits, J., Airiau, C., Hanifi, A., and Henningson, D., "Sensitivity analysis using adjoint parabolized stability equations for compressible flows," Flow Turbul. Combust., Vol. 65, 2000, pp. 183-210.

${ }^{39} \mathrm{Li}$, F. and Malik, M., "Mathematical nature of parabolized stability equations," R. Kobayashi (Ed.), Laminar-Turbulent Transition, Springer, 1994, pp. 205-212.

${ }^{40} \mathrm{Li}$, F. and Malik, M., "On the nature of the PSE Approximation," Theor. Comp. Fluid Dyn., Vol. 8, 1996, pp. $253-273$.

${ }^{41} \mathrm{Li}, \mathrm{F}$. and Malik, M., "Spectral analysis of parabolized stability equations," Compt. Fluids, Vol. 26 (3), 1997, pp. 279-297.

${ }^{42}$ Haj-Hariri, H., "Characteristics analysis of the parabolized stability equations," Stud. Appl. Math., Vol. 92, 1994, pp. 4153.

${ }^{43}$ Andersson, P., Henningson, D., and Hanifi, A., "On a stabilization procedure for the parabolic stability equations," J. Engng. Math., Vol. 33, 1998, pp. 311-332.

${ }^{44}$ Bagheri, S. and Hanifi, A., "The stabilizing effect of streaks on Tollmien-Schlichting and oblique waves: a parametric study," Phys. Fluids, Vol. 19, 2007, pp. 078103-1-078103-4.

${ }^{45}$ Mack, L. M., "Boundary Layer Stability Theory," Tech. Rep. 900-277, Jet Propulsion Lab., Pasadena, CA, 1969.

${ }^{46}$ Hermanns, M. and Hernández, J. A., "Stable high-order finite-difference methods based on non-uniform grid point distributions," Int. J. Numer. Meth. Fluids, Vol. 56, 2008, pp. 233-255.

${ }^{47}$ Paredes, P., Hermanns, M., Le Clainche, S., and Theofilis, V., "Order $10^{4}$ speedup in global linear instability analysis using matrix formation," Comput. Meth. Appl. Mech. Eng., Vol. 253, 2013, pp. 287-304. 
${ }^{48}$ Litton, D., Edwards, J., and White, J., "Algorithmic enhancements to the VULCAN Navier-Stokes solver," AIAA Paper 2003-3979.

${ }^{49}$ Andersson, P., Brandt, L., Bottaro, A., and Henningson, D., "On the breakdown of boundary layer streaks," J. Fluid Mech., Vol. 428, 2001, pp. 29-60.

${ }^{50}$ Paredes, P., Choudhari, M., and Li, F., "Nonlinear transient growth and boundary layer transition," AIAA Paper submitted for presentation at the AVIATION-2016 Meeting, Washington, D.C., June, 2016.

${ }^{51}$ Hiemenz, K., "Die Grenzschicht an einem in den gleichförmigen Flüssigkeitsstrom eingetauchten geraden Kreiszylinder," Dingl. Polytechn. J., Vol. 326, 1911, pp. 321-324.

${ }^{52}$ Schlichting, H. and K.Gersten, Boundary Layer Theory, Springer, 7th ed., 2000. 